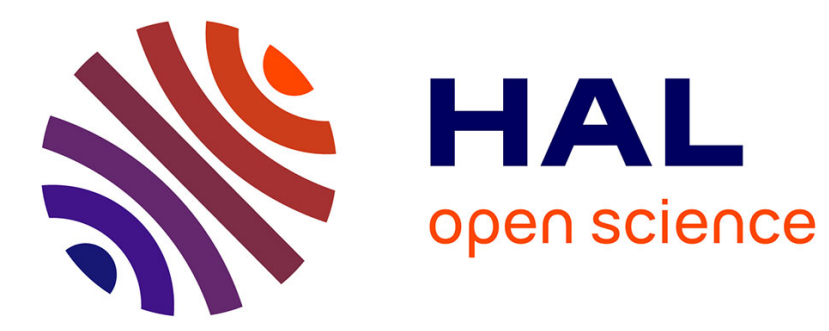

\title{
De la topicalité des adverbiaux détachés en tête de phrase
}

\author{
Michel Charolles
}

\section{To cite this version:}

Michel Charolles. De la topicalité des adverbiaux détachés en tête de phrase. Travaux de Linguistique: Revue Internationale de Linguistique Française, 2003, 47, pp.11-51. 10.3917/tl.047.0011. hal-00665853

\section{HAL Id: hal-00665853 \\ https://hal.science/hal-00665853}

Submitted on 2 Feb 2012

HAL is a multi-disciplinary open access archive for the deposit and dissemination of scientific research documents, whether they are published or not. The documents may come from teaching and research institutions in France or abroad, or from public or private research centers.
L'archive ouverte pluridisciplinaire HAL, est destinée au dépôt et à la diffusion de documents scientifiques de niveau recherche, publiés ou non, émanant des établissements d'enseignement et de recherche français ou étrangers, des laboratoires publics ou privés. 
Draft

CHAROLLES M., 2003, "De la topicalité des adverbiaux détachés en tête de phrase", in M. Charolles \& S.Prévost eds. Adverbiaux et topiques, Louvain la Neuve, Travaux de Linguistique, 47, 11-51.

\title{
DE LA TOPICALITE DES ADVERBIAUX DETACHES EN TETE DE PHRASE
}

\author{
Michel Charolles \\ Université de Paris III \\ UMR-CNRS 8094, LATTICE \\ ENS Ulm
}

Les expressions peu intégrées syntaxiquement figurant en zone préverbale sont plus ou moins prédestinées à fonctionner comme des thèmes ou des topiques ${ }^{1}$. C'est le cas en particulier des SN référentiels dans les constructions disloquées qui sont très courantes à l'oral en français. Le détachement d'un SN suivi de sa reprise sous la forme d'un pronom clitique facilite l'intégration des informations dans la mesure où celle-ci se fait en deux temps : mise en place d'un référent en relation avec le contexte précédent et ensuite expression des liens qu'il contracte dans la prédication. Ce principe de séparation de la référence et du rôle mis au jour par Lambrecht (1984) n'a pas d'équivalent avec les groupes adverbiaux détachés en position préverbale qui sont fréquents à l'écrit. On peut parler de topiques scéniques ou cadratifs mais ces appellations ne fournissent ni ne suggèrent aucune explication cognitive comparable.

Pour tâcher de comprendre quel bénéfice fonctionnel offrent ces constructions, il peut être intéressant d'aborder le problème en se mettant à la place du sujet qui doit produire ou traiter un énoncé au fur et à mesure qu'il l'émet ou le reçoit. C'est ce que nous faisons dans la première partie du travail qui suit en adoptant le point de vue du lecteur. Cette première investigation, fondée sur quelques phrases isolées, conduit à formuler des hypothèses sur les contextes amont et aval dans lesquels les adverbiaux détachés en tête de phrase semblent les mieux adaptés. Partant de là, nous présentons et discutons deux études sur corpus de Ramsay (1984) et de Ford \& Thompson (1986) portant sur les liens que les sous-phrases adverbiales antéposées entretiennent avec le discours précédent. Comme ces liens n'épuisent pas ceux qui sont attachés à la fonction de topique, nous présentons ensuite un article de Jacobs (2001) traitant des relations topique-commentaire. Dans la dernière partie qui prolonge la discussion de cet article, nous revenons sur les opérations d'adressage et de cadrage qui, dans la conception de Jacobs, constituent deux des quatre dimensions de cette relation. Sans remettre en cause le bien fondé de cette distinction, nous défendons l'idée que le cadrage implique une forme particulière d'adressage en ce qu'il fixe un critère pour la répartition des informations textuelles dans des blocs satisfaisant au critère spécifié par l'adverbial antéposé, à moins que le sujet de la proposition en tête de laquelle il apparaît ne soit postposé (cf. C.Fuchs \& N.Fournier, ce volume). En conclusion, nous insistons sur le fait que ce pouvoir confère aux adverbiaux cadratifs un rôle de premier plan dans la cohésion du discours. ${ }^{2}$

\footnotetext{
1 Dans la suite nous parlerons de topique. Ce choix est de pure convention : thème nous irait aussi bien.

2 Je remercie Anne Le Draoulec, Catherine Fuchs, Sophie Prévost, et Denis Vigier pour leurs commentaires, suggestions et remarques. Les erreurs sont miennes.
} 


\section{INCIDENCE DE LA PLACE DES ADVERBIAUX SUR LEUR INTERPRETATION}

Les travaux consacrés aux compléments circonstanciels (cf. Mélis (1983), Guimier ed. (1993), Guimier (1996), Leeman (1998) Molinier-Lelièvre (2000)) et aux constructions détachées (Combettes (1998) $)^{3}$ montrent que ces constituants peu intégrés syntaxiquement le sont à différents degrés. Les tests syntaxiques et sémantiques utilisés par les auteurs (possibilité d'effacement, mobilité, comportement sous focalisation, interrogation, négation, etc.) pour justifier les classements qu'ils proposent mettent en jeu des phrases achevées (en les rapportant à leur contexte d'usage), autrement dit des formes configurées dont la structure en particulier syntaxique est stabilisée. Cette façon de procéder, pour classique et nécessaire qu'elle soit, ne va pas sans soulever certaines difficultés, ne serait-ce que parce que les expressions potentiellement rectrices et, au premier chef les verbes, étant très souvent polysémiques, c'est la présence des compléments qui, dans un grand nombre de cas, permet de sélectionner l'acception et la grille argumentale correspondant à leur emploi et donc de statuer (a posteriori) sur le degré d'intégration desdits compléments dans la phrase. D'où l'intérêt de compléter les approches structurales de ce type par des investigations procédurales, notamment à propos de phénomènes dans lesquels la place occupée par les constituants est un paramètre essentiel, ce qui est le cas avec les constituants détachés en tête de phrase.

Pour illustrer ce point, considérons le début de phrase suivant :

(1) Paul a posé sa valise

Dans (1), le complément élimine une valeur du verbe (prendre la pose) dans laquelle celui-ci est intransitif et il sélectionne une acception de poser (déposer) qui appelle un argument supplémentaire, en l'occurrence un lieu cible. Le procès (de type achèvement) ne peut être conçu sans un complément de lieu, l'auditeur/lecteur s'attend à ce qu'on lui dise en quel endroit se trouve la valise à l'issue du procès comme dans :

(2) Paul a posé sa valise dans l'entrée.

Avec :

\section{(3) Paul a posé sa cravate}

l'auditeur/lecteur ne peut savoir si le verbe poser doit être entendu dans le sens (i) probablement proéminent - où il signifie déposer et où il appelle un lieu cible, ou bien s'il doit être compris dans l'acception (ii), où il n'appelle pas de locatif parce qu'il désigne un changement d'état (Paul s'est décravaté) ${ }^{4}$. Si on adjoint un complément de lieu à (3) :

(4) Paul a posé sa cravate dans l'entrée

son occurrence favorise plutôt l'interprétation (i), sans exclure toutefois l'interprétation (ii) où poser commute avec ôter. Dans l'interprétation (i), le complément de lieu occupe une position argumentale et indique l'endroit où se trouve l'objet. Dans l'interprétation (ii), il n'est pas sélectionné par le verbe et la construction ne localise pas l'objet : le SP a une incidence sur le sujet mais, dans la mesure où le changement d'état implique le verbe et le COD, il localise la relation prédicative dans son entier. Dans cette interprétation intraprédicative de (4), le complément de lieu est rhématique (foyer/focus) et il répond, comme dans la lecture (i), à la question : Où Paul a-t-il posé sa cravate? de la même façon que (2) répond à : Où Paul a-t-il posé sa valise?, quoique, dans (4), il ne soit pas un argument du verbe.

Dans (2), le complément occupant une position argumentale est difficilement antéposable:

\footnotetext{
3 Pour ne citer que quelques ouvrages sur le sujet.

4 Un complément comme (Paul a posé) les rideaux solliciterait une autre acception du verbe poser et exigerait d'autres commentaires.
} 
(5) ?? Dans l'entrée, Paul a posé sa valise.

L'énoncé est potentiellement récupérable (Paul a cessé de courir le monde) mais l'interprétation métaphorique où le verbe dénote un changement d'état est peu compatible avec le lieu indiqué (comparer avec : A son retour d'Afghanistan, Paul a posé sa valise). Par contre, l'antéposition du circonstant ne pose aucun problème avec (4) :

(6) Dans l'entrée, Paul a posé sa cravate.

La phrase impose une lecture du type (ii) dans laquelle poser est compris dans le sens de ôter. Ce qui est localisé dans (6), ce n'est cependant pas, comme dans l'interprétation intraprédicative (ii) de (4) (Paul a posé/ôté sa cravate dans l'entrée), le changement d'état de Paul, mais le fait que constitue ce changement d'état. La phrase ne répond pas à Où Paul a-t-il posé sa cravate? mais à : Que s'est-il passé dans l'entrée ? La question ne présuppose pas que Paul a posé sa cravate (i.e. s'est décravaté) dans un certain endroit à préciser, comme c'est le cas avec (4) dans la lecture (ii). Elle présuppose qu'il s'est passé quelque chose dans l'entrée et que c'est ce quelque chose qui a besoin d'être spécifié. Le SP est extraprédicatif, il est hors de la portée de la question, il en est le point de départ, pas l'objet, alors qu'avec (4) dans l'interprétation (ii), bien que le complément ne soit pas argumental, il en demeure le foyer, et il en irait de même avec la négation.

Quand on compare (6) et (4) dans l'interprétation où le verbe dénote un changement d'état, on s'aperçoit également que le complément prend, dans (6), une valeur temporelle qui est absente de (4). L'énoncé est compris comme signifiant une fois/sitôt dans l'entrée / dès l'entrée, Paul s'est décravaté. Cet effet est facilité par le fait que le $\mathrm{N}$ entrée est un déverbal évoquant un parcours et donc une suite d'actions chronologiquement ordonnées. Mais la présence d'un $\mathrm{N}$ déverbal de ce type n'est pas une condition nécessaire pour l'apparition de cette valeur temporelle. Une phrase comme : Dans la voiture, Paul a posé sa cravate, se prêterait tout autant à une lecture temporelle (Une fois dans la voiture).

Ce déplacement de sens, qui est très courant avec les circonstants de lieu antéposés ${ }^{5}$, se traduit par une forme de coalescence des valeurs spatiales et temporelles. Certaines constructions comportant des SP en avec se prêtent à des déplacements encore plus poussés. Par exemple, dans (7), que nous empruntons à J.J.Franckel \& D.Paillard (1999, p.280) :

(7) Paul se promène avec son chien.

l'intégration du SP postposé est très facile même si, comme l'écrivent J.J.Franckel \& D.Paillard (1999, p.281), "il est difficile de considérer le SN introduit par avec comme un argument de se promener". L'antéposition :

(8) Avec son chien, Paul se promène.

prend une valeur conditionnelle (Quand il a la garde de son chien, Paul est bien obligé de se promener) en raison du caractère habituel de (8), lecture qui serait bloquée si le verbe était au passé composé :

(9) *Avec son chien, Paul s'est promené (hier).

Dans (7), la préposition a une valeur comitative forte (Paul et le chien se promènent). Par contre, dans (8), cette valeur est atténuée dans la mesure où elle met en avant Paul qui est au centre de l'événement. Cette valeur peut même disparaître comme dans :

(10) Avec son chien, Paul doit rentrer déjeuner chez lui.

où le chien n'est plus du tout impliqué dans le déplacement et où le complément n'est plus postposable ${ }^{6}$ sans modification du sens :

\footnotetext{
5 Cf. Huumo (1996).

${ }^{6}$ La bizarrerie de Les portes s'ouvrent avec Paul relevée par J.J.Franckel \& D.Paillard (1999, p.280) tient sans doute pour partie au fait que le complément tend à être intégré ce qui favorise à la suite de les portes s'ouvrent une lecture instrumentale qui est stéréotypique mais incompatible avec un SN humain.
} 


\section{(11) Paul doit rentrer déjeuner chez lui avec son chien.}

Dans :

(12) Avec son chien, Paul est insupportable.

compris dans le sens il nous rebat les oreilles avec son chien, un pas de plus est franchi : le SP prend une valeur causale et énonciative (le chien est cause du fait que Paul en parle tout le temps), valeur que l'on trouve couramment à l'oral dans les emplois du genre de :

(13) Avec mon mari (de malade), je suis clouée à la maison.

Les déplacements de sens que l'on vient de relever s'apparentent à une forme de désémantisation ce qui suggère que les adverbiaux antéposés jouent un autre rôle que les circonstants ordinaires. Pour préciser ce point, considérons :

(14) La cheminée fumait sur le toit.

Le complément, bien que non argumental, a tendance, comme dans (4), à être rattaché à la prédication : on comprend que la cheminée dégage de la fumée en direction du toit. Cette lecture est relativement coûteuse car elle oblige à forcer le verbe pour expliciter l'objet interne et faire paraître un verbe support compatible avec un complément de lieu. Elle contraste avec celle, beaucoup plus naturelle, au moins si l'on s'en tient à la phrase isolée, qui accompagne :

(15) Sur le toit, la cheminée fumait.

où le verbe reste intransitif. Dans (15), l'antéposition du circonstant se traduit, comme dans (4), par un infléchissement de sa valeur spatiale : l'énoncé est affecté à un sujet percevant qui relève que la cheminée fume à la suite d'une sorte de parcours des différentes parties de la maison, ce qui a pour effet de situer cette notation dans une chronologie, à défaut de lui conférer une valeur à proprement parler temporelle ${ }^{7}$. L'antéposition du circonstant de lieu impose aussi certaines restrictions sur le contexte précédent et suivant. (14) n'exige aucun contexte particulier si ce n'est une situation à même de rendre son énonciation plausible comme par exemple :

(16) Il était impossible d'aménager une terrasse à la place des combles. La cheminée fumait sur le toit.

(15) par contre est difficilement recevable dans le même contexte :

(17) ?? Il était impossible d'aménager une terrasse à la place des combles. Sur le toit, la cheminée fumait.

Avec (15), il faut par contre que, d'une façon ou d'une autre, l'antéposition soit justifiée ou au minimum préparée ainsi que dans :

(18) Paul regarda la maison. Sur le toit, la cheminée fumait.

(14) est également possible dans le même contexte :

(19) Paul regarda la maison. La cheminée fumait sur le toit.

sauf que (19) annonce plutôt un relevé des choses qui sont en mauvais état dans la maison :

(20) Paul regarda la maison. La cheminée fumait sur le toit. Les fenêtres étaient en très mauvais état. ...

pendant que (17) anticipe un parcours des autres parties de la maison :

(21) Paul regarda la maison. Sur le toit, la cheminée fumait. Au premier étage, une pièce était éclairée. ....

Lorsque le circonstant de lieu précède la prédication, il ne se contente donc pas, comme nous l'avons relevé jusque-là, de porter sur l'état de choses qu'elle dénote, il ne se contente même pas d'imputer cet état de choses à un sujet percevant, il impute cet état de choses à un sujet parlant qui organise son propos à partir d'un critère, en l'occurrence, dans (15), d'un critère

\footnotetext{
${ }^{7}$ Le contraste entre (14) et (15) se traduirait probablement à l'oral dans les emplois en situation immédiate où le locuteur, pour attirer l'attention de l'interlocuteur, choisirait plutôt Regarde, il y a la cheminée qui fume sur le toit pour "dire" (14) et Regarde, sur le toit, il y la cheminée qui fume pour "dire" (15).
} 
spatial. Ce critère a besoin d'être justifié en amont et on s'attend à ce qu'il soit exploité au mieux en aval, ce qui est le cas dans les enchaînements contrastifs du type de (21).

Les adverbiaux antéposés n'ont pas besoin d'être détachés par une virgule pour être extraprédicatifs (même si cela est le plus souvent le cas ${ }^{8}$ ) : leur emplacement suffit dans les propositions à sujet antéposé (cf. C.Fuchs \& N.Fournier, K.Lahousse, ce volume). Lorsqu'ils sont postposés, on pourrait par contre penser que leur détachement à la suite d'une virgule suffit à empêcher leur intégration à la prédication et pour qu'ils fonctionnent comme les extraprédicatifs. Or, quand on insère une virgule avant le SP dans (14) :

(22) La cheminée fumait, sur le toit.

l'interprétation qui s'impose est plutôt du type :

(23) La cheminée fumait, mais elle fumait sur le toit.

avec rattachement après coup à la prédication. (22) simule l'oral, avec intonation plate sur l'adverbial : le locuteur répartit l'information en deux temps, il asserte d'abord que la cheminée fume et ensuite il s'appuie sur cette information (partagée) pour ajouter une restriction (Chafe 1994, Lambrecht 1994, à par.a et b). L'exemple (22) se prête également à une lecture extraprédicative proche de celle de (15). Dans cette lecture, le fait que la cheminée fume est compris comme un indice du fait que (par exemple) la maison est occupée. (15) invite très facilement à ce genre d'interprétation, comme on le voit bien avec (21) qui enchaîne sur une phrase faisant allusion à un autre signe de la présence d'occupants. Avec (22), la lecture extraprédicative est moins facile du fait du rajout du circonstant : on ne voit pas quelle motivation pourrait conduire le locuteur à préciser après coup que c'est sur le toit que cet indice se manifeste, vu que les cheminées se trouvent habituellement en cet endroit. Cette différence entre (15) et (22) s'explique assez bien. Dans (22), l'information spatiale détachée en tête n'est pas liée seulement au fait mentionné dans la phrase qui suit mais à un fait externe, à savoir, comme on l'a vu, à la démarche d'un sujet percevant qui scrute la maison. Ce sujet percevant appelé par la construction et généralement évoqué dans le contexte précédent, fournit d'emblée un support de "point de vue", ce qui facilite une lecture "argumentative" du fait exprimé ensuite. La récupération de (15) sur ce mode n'est pas exclue mais elle est plus coûteuse dans la mesure où la construction ne met pas d'emblée l'accent sur un sujet percevant.

Avec (4) (Paul a posé sa cravate dans l'entrée) le détachement du circonstant :

(24) Paul a posé sa cravate, dans l'entrée.

est réintégré dans les deux lectures possibles : (i) par spécification après coup du complément argumental implicité (Paul déposé sa cravate quelque part, et ce quelque part est l'entrée), et (ii) par rattachement à la prédication (Paul s'est décravaté mais il s'est décravaté dans l'entrée, sous-entendu : personne ne l'a vu). Dans un cas comme dans l'autre, le détachement en final est plutôt compris comme exprimant une précision a posteriori, interprétation qui est exclue lorsque le complément figure en tête de phrase car il est impossible de rectifier par avance une information que l'on n'a pas encore énoncée. Même dans la lecture (ii) où la rectification met en jeu un complément extraprédicatif, celui-ci, quoique demeurant extraprédicatif, n'a pas exactement les mêmes pouvoirs que lorsqu'il est détaché en tête. À la suite de (6) (Dans l'entrée, Paul a posé sa cravate) où le complément est détaché en position frontale, on peut parfaitement avoir :

(25) Dans l'entrée, Paul a posé sa cravate. Des invités sont arrivés. Une personne a fait les présentations.

alors, qu'avec (24), la même suite passe moins bien :

\footnotetext{
${ }^{8}$ Cf. Chafe (1984)
} 
(26) ? Paul a posé sa cravate, dans l'entrée. Des invités sont arrivés. Un personne a fait les présentations.

La séquence (26) paraît en effet moins cohérente : le locuteur après avoir noté le changement d'état de Paul, ajoute une précision concernant l'endroit où ce changement d'état est intervenu. Cette précision additionnelle ne remettant pas en cause le fait que le locuteur a pour intention de rapporter ce qu'a fait Paul, s'il est ensuite fait allusion à d'autres événements sans rapport avec cette personne, on inférera que le locuteur rapporte des faits qui se sont déroulés ensuite, mais pas nécessairement dans le même lieu (les invités ont pu arriver par le jardin, les présentations ont pu avoir lieu dans le salon où se trouve le locuteur). A l'inverse, la séquence (25) ne suscite aucune impression d'incohérence, on comprend très naturellement que le locuteur rapporte une série d'événements qui se sont tous déroulés dans l'entrée, avant d'évoquer, le cas échéant, ce qui a pu se passer dans un autre endroit ( $A u$ salon, ...). Dans (26), les événements dénotés par la seconde et la troisième phrases ont aussi tendance à être compris comme successifs alors que dans (25) ils sont simultanés. Même s'il est difficile d'imaginer que dans (26) les actions mentionnées se passent exactement au même instant, le circonstant de lieu étant détaché en tête de la première phrase prend une valeur temporelle (cf. ci-avant) qui tend à servir également de repère pour les phrases suivantes ${ }^{9}$.

Les analyses présentées jusque-là ne mettent en jeu que des SP mais elles valent également pour les subordonnées adverbiales. Pour illustrer ce point rapidement, nous nous limiterons aux constructions conditionnelles du type de :

(27) Paul viendra si Marie l'invite.

Dans (27), la subordonnée n'occupe pas une position argumentale (privilège des si percontatifs/d'interrogation indirecte). Le fait qu'elle apparaisse après la principale favorise cependant une lecture intraprédicative dans laquelle elle est le foyer de la phrase. Dans cette lecture, (27) répond à la question A quelle condition Paul viendra-t-il ? et la négation ne porte ni sur la protase ni sur l'apodose mais sur leur relation (Paul ne viendra pas si Marie l'invite mais si Jean l'invite). Le détachement en final après une virgule :

(28) Paul viendra, si Marie l'invite.

tend, comme précédemment, à maintenir cette interprétation, la subordonnée spécifiant après coup à quelle condition Paul viendra. L'antéposition de la conditionnelle :

(29) Si Marie invite Paul, il viendra.

sélectionne clairement l'interprétation extraprédicative où la conditionnelle prend une valeur différente : le locuteur pronostique que Paul viendra en se plaçant dans l'hypothèse où Marie l'invite. Dans cette lecture, le foyer est la principale : (29) répond à la question Que va-t-il se passer si Marie invite Paul? et la négation (il est inexact que si Marie invite Paul il viendra) ne remet pas en cause la protase. Le fait que l'on puisse dire :

(30) Paul ne viendra pas (,) si Marie l'invite

dans l'intention de signifier que Paul ne viendra pas dans l'hypothèse où Marie l'invite, suggère qu'il en va de même avec (27) et (28) qui n'excluent donc pas une lecture extraprédicative. Hormis cette différence, le comportement des subordonnées adverbiales, semble très proche de celui des SP.

Dans (29), l'hypothèse exprimée par la protase au présent de l'indicatif est envisagée comme une potentialité, avec le passé composé :

(31) Si Marie a invité Paul, il viendra.

cette hypothèse est, sinon avérée, au moins momentanément prise en charge par le locuteur. Dans (31), la protase est quasi échoïque (si, comme tu le dis, Marie a invité Paul) et donc

\footnotetext{
${ }^{9}$ Ce point est discuté dans Terran (2002) et Le Draoulec et Péry-Woodley (à par.). Sur la thématisation des compléments temporels cf. Berthonneau (1987)
} 
reliée au discours précédent. Cet attachement au contexte amont est peut-être moins sensible dans (29), mais l'antéposition de la protase au présent va aussi de pair avec un contexte dans lequel il est question d'inviter ou non Paul, alors que (27), dans la lecture intraprédicative, n'a pas besoin d'être annoncé par une alternative. (27) pourrait figurer en tête d'une nouvelle :

(32) "Paul viendra si Marie l'invite" se disait Sophie. Il faut que je la persuade de lui envoyer un carton.

Alors qu'attaquer avec (29) :

(33) "Si Marie invite Paul, il viendra" se disait Sophie. Il faut que je la persuade de lui envoyer un carton.

semblerait moins naturel ${ }^{10}$. On attendrait plutôt :

(34) "Est-ce que Marie va inviter Paul ?" se demandait Sophie. Si elle l'invite, il viendra, alors il faut que je la persuade de lui envoyer un carton.

Des observations qui précèdent, il ressort que l'antéposition des adverbiaux a des conséquences importantes sur leur traitement. Contrairement aux adverbiaux postposés qui ont tendance à être rattachés à la prédication, les adverbiaux antéposés résistent à tout forçage dans ce sens. Ils fixent un critère sémantique par rapport auquel l'information véhiculée par la phrase en tête de laquelle ils apparaissent doit être envisagée. Ce critère a besoin d'être motivé par le contexte précédent qui doit justifier la dimension des états de choses mise en avant. Intervenant avant que la relation prédicative ne soit installée, ce critère vaut pour la prédication qui suit, mais il est également disponible pour d'autres qui pourraient apparaître dans la suite, ce qui fait qu'il peut être exploité pour la répartition des informations à l'échelle du texte. Nous reviendrons sur ce point dans la quatrième partie, mais auparavant nous allons présenter quelques études dans lesquelles ce type de fonctionnement a été rapporté au fait que les adverbiaux antéposés sont des topiques.

\section{II . LES ADVERBIAUX ANTEPOSES COMME TOPIQUES PAR L'AMONT}

\subsection{Deux études sur corpus ${ }^{11}$}

Les études de Ramsay (1984) et Ford et Thompson (1986) sont consacrées au fonctionnement des subordonnées adverbiales en if (et when pour la première) dans des textes écrits et des échanges oraux (pour la seconde). Les auteurs partant des analyses de Haiman (1978), nous allons en dire quelques mots pour commencer.

Pour défendre l'idée annoncée par le titre de son article, à savoir "Conditionals are topics", Haiman (1978) s'appuie sur des exemples "classiques" mais aussi et surtout sur des arguments morphologiques tirés de la comparaison entre les langues. Il rapporte ainsi qu'en hua il existe trois façons d'exprimer les conditionnelles : les "medials", les interrogatives et les constructions spécifiquement conditionnelles qui sont marquées par une désinence personnelle $(\mathrm{ma})$ et une particule connective $(\mathrm{mo})$. La désinence personnelle ma que l'on retrouve dans certaines constructions "medials" et dans les relatives signale que la proposition est "présupposée", quant à la particule connective mo, elle indique que le SN auquel elle est attachée va faire l'objet d'une prédication, autrement dit, d'un propos, ce qui suggère que la protase fonctionne comme un topique. De même, relève Haiman, en turc, le suffixe marquant les conditionnelles $-S A$ signale, quand il est appliqué à un $\mathrm{SN}$, que celui-ci est un topique

\footnotetext{
${ }^{10}$ Même avec un pronom cataphorique : Si Marie l'invite, Paul viendra.

${ }^{11}$ Pour une étude sur corpus des compléments circonstanciels en français cf. Guimier ed. 1993, et notamment pour les compléments antéposés, dans ce volume : Le Querler.
} 
contrastif (pour ce qui est/quant à) et il en va semblablement avec la particule kung du tagalog qui est un topicalisateur.

Le fait que les conditionnelles et les topiques soient marqués de la même façon dans un grand nombre de langues typologiquement différentes peut paraître étonnant mais, dit J.Haiman, il s'explique très bien. Pour ce faire, inutile de chercher à réduire la connexion entre la protase et l'apodose à une relation causale ou autre, il faut aborder les conditionnelles en termes de négociation des savoirs partagés :

"A conditional clause is (perhaps only hypothetically) a part of the knowledge shared by the speaker and his listener. As such it constitutes the framework which has been selected for the following discourse"

Cette position, rappelle Haiman, était déjà celle de Jespersen (1940) qui voyait dans les conditionnelles un condensé de mini séquences conversationnelles mettant en scène une séquence question-réponse. L'exemple de Jespersen que reprend Haiman est intéressant, il consiste à faire valoir qu'une conditionnelle comme : S'il vient, je reste simule une sorte de dialogue dans lequel le locuteur, après avoir demandé fictivement à son interlocuteur Est-ce qu'il va venir ? et obtenu de lui une réponse supposée affirmative, s'appuie sur cette réponse pour énoncer l'apodose. Semblablement, relève Haiman, les conditionnelles bi-négatives comme If Hitler was a military genius, I'm a monkey's uncle ou incohérentes comme If Hitler is a military genius, London is in England jouent sur les savoirs supposés partagés à propos à la fois de la protase (si, comme tu le dis) et de l'apodose (de son caractère notoirement faux ou sans rapport).

Haiman revendique une conception du topique dans laquelle celui-ci fait allusion à une information connue ou ancienne (i.e. déjà mentionnée dans le discours) ${ }^{12}$. Les topiques contrastifs ( $\mathrm{SN}$ accentués, ou introduits à la suite de quant à/ pour ce qui est de, etc.) qui apportent une information nouvelle, ne constituent pas une objection à cette conception, dans la mesure où, dit Haiman, ils ne font que sélectionner une entité prélevée sur un ensemble déjà évoqué ou sollicitent explicitement l'accord de l'interlocuteur. A quoi il ajoute, en s'appuyant sur Kuno, que les topiques contrastifs sont marqués en japonais par la particule wa qui est utilisée pour les expressions renvoyant à une information déjà communiquée (cf. D.Klingler \& M.Yates, ce volume). La définition des topiques en termes de connu (vs nouveau), explique Haiman, est préférable à celle consistant à y voir ce dont le locuteur veut parler (aboutness). Si cette définition des topiques était fondée, on devrait s'attendre à ce qu'ils soient systématiquement repris (anaphorisés) dans le commentaire (comme dans les constructions disloquées), ce qui n'est pas le cas, sans compter que, ajoute Haiman, les topiques ne sont pas nécessairement ce dont on parle, ou ce à propos de quoi est la phrase. Ils en constituent parfois seulement le "framework" qui, comme dit Chafe (1976: 50), " limits the applicability of the main predication to a certain restricted domain". Dans le même esprit, Haiman s'appuie, à propos des conditionnelles, sur les analyses de Ducrot (1972) pour qui :

"une proposition du type si $p, q$ n'a pas pour signification première "p est cause de q", ni "p est condition de q" (bien qu'elle puisse servir à indiquer ces relations). Sa valeur fondamentale est de permettre la réalisation successive de deux actes illocutoires : $1^{\circ}$ demander à l'auditeur d'imaginer "p", $2^{\circ}$ une fois le dialogue introduit dans cette situation imaginaire, y affirmer "q"" (p.168).

A quoi, on pourrait rajouter de Cornulier (1985) qui, dans un esprit très proche, défend que ""Si P, Q" signifie à peu près "Dans le ou les cas où $\mathrm{P}, \mathrm{Q}$ "" (p.58)

\footnotetext{
${ }^{12}$ Cette conception, comme on sait, a fait l'objet de nombreuses discussions dans la suite, pour une critique cf. notamment Reinhart (1981).
} 
Les articles de Ramsay (1984) et de Ford \& Thompson (1986) prolongent directement l'étude de Haiman avec une perspective discursive absente chez ce dernier. Cette perspective est ouvertement affichée, notamment par Ford et Thompson qui regrettent que "Haiman (...) misses the chance to use discourse evidence for his characterization of the work performed by conditionals" (p. 353) car, disent-elles, il est nécessaire d'étudier le fonctionnement des constructions en if à ce niveau si on veut savoir "how conditionals are used rather than how we think they are used" (p. 354).

L'étude de Ramsay sur les "Preposed and postposed "if" and "when" clauses" est fondée sur un corpus de 109 "if clauses" (IC) et 95 "when clauses" (WC) extraites d"'une nouvelle policière ${ }^{13}$. Dans ce corpus, le pourcentage des IC et WC antéposés est plus élevé que celui des IC et WC postposés (pratiquement le double). Les WC en position intiale sont plus fréquentes que les IC dans les segments affectés au narrateur, mais cette tendance s'inverse dans les propos rapportés. Pour évaluer le lien avec le discours antérieur, Ramsay compte :

- a) le nombre de propositions tensées ("gaps") séparant les SN sujets des IC et WC et des principales avec un SN antérieur coréférentiel. La distance est évaluée à l'intérieur du discours soit du narrateur soit des personnages ${ }^{14}$. De ce premier décompte, il ressort que plus de $90 \%$ des IC postposées comportent une expression coréfèrant avec une autre se trouvant dans la phrase immédiatement précédente, le plus souvent la principale. Avec les WC postposées, le pourcentage est plus faible, surtout dans les paroles rapportées. Par contre, les IC et WC antéposées coréfèrent plus souvent avec une expression se trouvant dans une proposition plus éloignée en amont, cette tendance étant plus marquée avec les IC qu'avec les WC.

- b) le nombre de propositions tensées séparant les SN sujets des principales (lorsque celles-ci comportent des IC et WC antéposées) avec leur antécédent en amont Quand le sujet de la principale coréfère avec une expression d'une phrase antérieure, le plus souvent $(70 \%$ à $90 \%$ des cas) c'est avec une expression de la subordonnée antéposée qui précède, et il n'y a pas de différence entre le comportement des IC et des WC. Dans la moitié des cas, la principale introduit un référent nouveau..

- c) le nombre de propositions tensées séparant chaque IC et WC avec la proposition antérieure nécessaire pour les comprendre (et pas seulement leur sujet). Cette mesure révèle que plus de $80 \%$ des IC postposées ont une "portée" en amont de 1 à 3 propositions pendant que les IC antéposées ont une portée plus importante, notamment dans les propos affectés à des personnages où la dispersion est importante et peut dépasser les 20 propositions. Les WC postposées se comportent à peu près comme les IC mais leur moyenne d'éloignement est un peu plus faible.

Sur la base de ces observations, Ramsay conclut que les "preposed IC and WC appear to be thematically linked to the main clause as well as, and even more frequently, to the preceding discourse. Postposed clauses, on the other hand, appear to have a much higher referential continuity to the main clause" (p.402) et ajoute que : "the thematic link of a preposed clause is much wider than that of a postposed clause" (p. 402)

\footnotetext{
${ }^{13}$ Ces décomptes excluent les IC et WC "complétifs" (to know when/if), les IC et WC relatifs (the moments when), les constructions en as if, if only et les "commentaires" du type if that doesn't sound too pompous qui n'ont (en général) pas de conséquent ou qui appraissent en fin de P.

${ }^{14}$ Les SN sujets nouveaux (ne se rattachant à aucune mention antérieure) n'apparaissent pas dans le décompte, de même Ramsay ne compte pas les pronoms personnels de première et seconde personnes, les pronoms indéfinis ou non référentiels, ni les $\mathrm{SN}$ abstraits ou génériques.
} 
L'étude de Ford \& Thompson (1986) ne porte que sur les conditionnelles Elle est fondée sur un corpus écrit composé d'un texte ("narratif") rapportant les tentatives d'apprentissage de la langue des signes à un chimpanzé, d'un ouvrage ("technique") de mécanique automobile et d'un essai ("argumentatif") de B.Russell, ainsi que d'un corpus oral constitué de la transcription de deux cours à l'université, d'un exposé d'étudiant et d'une conversation sur un sujet politique imposé. Le nombre d'occurrences de conditionnelles est nettement plus important que chez Ramsay (respectivement 490 contre 109) ${ }^{15}$.

Ford et Thompson dénombrent plus de conditionnelles en if à l'oral qu'à l'écrit $(7,2$ contre 4,6 pour 1000 mots). Le pourcentage des if à l'initiale est d'environ $80 \%$ dans les deux corpus contre $20 \%$ en final. Cette différence est telle que l'antéposition de la subordonnée constitue, disent Ford et Thompson, le cas non marqué ${ }^{16}$, ce qui étaye déjà l'analyse de Haiman. A la différence de Ramsay, Ford et Thompson ne cherchent pas à repérer précisément le point d'ancrage des conditionnelles antéposées dans le discours précédent ni à évaluer sa distance d'avec celui-ci. Elles proposent une typologie des configurations d'emploi des conditionnelles antéposées qui sont les plus attestées dans leur corpus. Ces configurations sont les suivantes :

a) les emplois (dits "exploring") du type "X. Assuming $X$, then $Y^{\prime \prime}$ dans lesquels la conditionnelle reprend un constituant déjà mentionné et sert de cadre pour l'apodose,

b) les emplois ("contrasting") du type "X. (But) if not $X$, then $Y^{\prime \prime}$ qui suivent le même schéma que les précédents, sauf que la conditionnelle contraste avec une assertion précédente (la contrefactualité étant une forme de contraste),

c) les emplois ("particular cases") du type "Generealization. (For exemple) if $X$ then $Y^{\prime \prime}$ où la protase illustre un cas particulier annoncé par une assertion de portée générale,

d) les emplois ("assuming") du type $X$. If Option $Y$, then $Z$ dans lesquels la protase "opens a new possibility whose consequences are to be explored" (p.358)

Dans le corpus écrit, $57 \%$ des conditionnelles sont du type exploring (jusqu'à près de $80 \%$ dans le texte technique), les emplois du type contraste et cas particuliers obiennent le même score de 17,5\% (ils sont à peu près également répartis dans les trois types de textes considérés, sauf pour les emplois du type cas particuliers qui sont moins représentés dans les textes techniques), enfin, les emplois dits "assuming" qui introduisent une option inédite ont un pourcentage de seulement $8 \%$ en moyenne ( $2 \%$ dans l'ouvrage de mécanique). Ce dernier pourcentage, lorsqu'on le rapporte au total de ceux obtenus avec les trois premières configurations $(92 \%)$ où le lien avec le contexte est très clair, conforte lui aussi l'analyse de Haiman.

A l'écrit, seulement $23 \%$ des subordonnées en if apparaissent en position finale. Les facteurs susceptibles d'expliquer ce choix "marqué" sont divers. Ford et Thompson relèvent que les conditionnelles ont tendance à être postposées lorsqu'elles sont dans une nominalisation, une infinitive ou une relative et où elles apportent plutôt des précisions sans rapport avec le topique de l'apodose. Autre cas favorable à la postposition : lorsque la conditionnelle apporte une information "lourde" qui ne fait pas partie des connaissances partagées ou évoquées et qui, de ce fait, passerait mal en position initiale où elle serait

\footnotetext{
${ }^{15}$ Ford \&Thompson excluent de leur relevé les conditionnelles tronquées du type "if possible", les conditionnelles précédées de "only", "even" et "unless", ainsi que celles qui sont en incise postverbale (ex. "if it occurs")

${ }^{16}$ Fait déjà relevé, notent Ford \& Thompson, par Greenberg et Comrie, cf. également Diessel (2001)
} 
présentée comme déjà connue. Enfin, Ford et Thompson relèvent que les conditionnelles qui ont une taille importante ont également plus tendance à être postposées que celles qui sont plus courtes. Dans le corpus oral, les pourcentages de conditionnelles antéposées sont globalement les mêmes qu'à l'écrit et elles sont le plus souvent reliées au contexte précédent, mais les chiffres sont légèrement inférieurs dans chaque rubrique du fait de l'existence d'une catégorie supplémentaire où elles expriment une requête de politesse (environ 10\% des cas ). Dans le corpus oral, on retrouve, pour les conditionnelles en finale, les mêmes tendances qu'à l'écrit, avec en plus : des if d"'afterthoughts", des if restrictitfs et des if à la suite d'une question ou d'une autre conditionnelle, cas dans lesquels le lien avec le contexte immédiatement précédent est très étroit.

Ford et Thompson concluent en soulignant qu'une "non-initial if clause qualifies an associated proposition, but it does not display as clear a connection with preceding or subsequent discourse " (p.370), alors que les conditionnelles antéposées entretiennent des relations étroites avec le discours précédent.

\subsection{Discussion}

Les subordonnées adverbiales ayant un sujet propre, le lien avec le contexte amont qui fait qu'on les considère comme des topiques se résume très souvent au fait qu'elle comportent un SN coréfèrant avec un constituant de la phrase précédente ou d'une phrase proche. Les cas de ce type abondent :

(35) "La phase vasculaire est caractérisée par une réduction brutale du débit sanguin cérébral au niveau de la zone en aval de l'occlusion. Cette zone est bordée d'une région, appelée pénombre, qui présente une réduction plus faible du débit sanguin cérébral : sa capacité à survivre dépend de la durée de l'occlusion . Si celle-ci $\boldsymbol{i}_{\boldsymbol{i}}$ se prolonge, la pénombre évolue lentement, parfois en plusieurs jours, vers une lésion totale." (La Recherche)

La relation n'est cependant pas toujours aussi évidente. Dans l'extrait suivant :

(36) "(...) les obstétriciens s'interrogent: les césariennes diminueraient-elles la contamination des nouveau-nés qui ont échappé au virus in utero? Si cette voie de recherche semble prometteuse, son efficacité devra être confirmée ainsi que celle de l'AZT destiné à protéger le nourrisson contre une infection potentielle." (La Recherche)

le SN démonstratif sujet de la protase s'appuie sur la question qui précède mais le fait que les spécialistes s'interrogent sur la possibilité que les césariennes diminuent la contamination des nouveaux nés n'évoque pas directement "une voie de recherche". Le sujet de la subordonnée ne renvoie pas à un référent précédemment introduit, on peut tout au plus parler, comme Haiman, de relation "résomptive" ou dire, comme Ford \& Thompson, que "In English, a conditional brings a complex referent - explicit background information expressed in a clause - into the discourse." ( p.370) ${ }^{17}$. Cette relation ne suffit cependant pas pour que l'on considère que le SN démonstratif fait allusion à une information "connue" au moment où il est employé. Le lien est, au mieux, inférable (Prince 1981) et le référent "semi-actif" (Chafe 1994, Lambrecht 1994) dans la représentation du lecteur au sens où, effectivement, à la lecture du $\mathrm{SN}$, donc a posteriori, on est prêt à admettre que les interrogations des obstréticiens soient associées aux voies de recherche qu'ils explorent et notamment à celle mentionnée. Lorsque le sujet de la conditionnelle est un indéfini comme dans :

(37) "Avec 135 millions de cas dans le monde en 1998, et 300 millions en 2025 selon

\footnotetext{
${ }^{17} \mathrm{Cf}$. Ramsay qui inclut dans son relevé (cf. c), la recherche d'une phrase antérieure susceptible de servir d'accroche à la subordonnée conditionnelle.
} 
les estimations de l'OMS, le diabète est de loin la plus répandue des maladies associées au sucre. Si des facteurs génétiques entrent en jeu, c'est surtout notre mode de vie qui explique l'explosion du diabète dans les pays occidentaux comme la France, dont au moins $2 \%$ de la population est déjà concernée." (La Recherche)

le lien avec un référent déjà mentionné dans le contexte est exclu (l'idée que des facteurs génétiques puissent jouer un rôle dans le diabète n'est pas activé par ce qui précède). Il n'empêche que la conditionnelle s'articule bien avec la phrase précédente et que son apparition n'a rien d'incongrue. Cette articulation passe par un processus interprétatif relativement complexe qui tient au sens instructionnel de si. L'énonciation d'une conditionnelle implique que le rédacteur et le lecteur s'accordent (provisoirement) pour sélectionner un état de choses (spécifié par la protase) sur un ensemble d'états de choses supposé accessible dans le contexte, et cela à la seule fin d'accéder à un nouvel état de choses qui est exprimé par l'apodose ${ }^{18}$. Ce fonctionnement s'applique parfaitement à (37) : le rédacteur, après avoir asserté que le diabète est une maladie très répandue, présume que le lecteur a en tête l'idée qu'un certain nombre de raisons doivent expliquer ce fait. Même si ce n'est pas le cas, l'énonciation de la protase devrait suffire à la fois à activer l'idée qu'il existe des explications à cet état de choses et à autoriser le prélèvement d'une explication déterminée, à savoir l'incidence des facteurs génétiques sur le déclenchement de la maladie. Tout en mentionnant le rôle de ces facteurs, l'auteur de (37) ne présente cependant pas ceux-ci comme décisifs, ce qui suggère qu'il prend ses distances avec ceux qui seraient enclins à les considérer comme tels, d'où l'apparition d'une valeur concessive qui pourrait être explicitée à l'aide de même si. Dans :

(38) La présence d'artefacts de pierre taillée et l'apparition progressive de comportements techno-économiques différenciés et structurés dans l'espace évoquent l'émergence de ce que les ethnologues appellent la culture. Néanmoins, plusieurs difficultés apparaissent immédiatement à cette évocation. D'abord, le foisonnement des espèces rend quasi impossible l'identification des hominidés qui ont fréquenté les sites de vie décrits (voir l'encadré : "Des acteurs si nombreux ») : toute comparaison rigoureuse entre productions matérielles et formes biologiques est hors de portée. En outre, dans les premières phases de développement des hominidés, le parallélisme semble étroit entre l'évolution biologique, notamment l'augmentation de la taille $d u$ cerveau, et l'évolution que nous avons cru déceler dans les comportements techno-économiques et écologiques. Si la culture est la part de variation des comportements à l'intérieur d'une espèce, nous sommes aujourd'hui totalement démunis pour en rendre compte.(La Recherche)

la sélection d'une définition particulière de la culture s'articule avec l'allusion (au début du passage) au fait que les ethnologues ont une certaine conception de celle-ci. En évoquant "ce que les ethnologues appellent la culture", le rédacteur donne à penser qu'il y a d'autres conceptions de celle-ci (d'autant qu'il relève les insuffisances de la conception en question) et cela suffit à motiver le fait qu'il en introduise une faisant le lien entre évolution biologique et comportement, quitte à dire ensuite qu'elle ne résout pas le problème.

Cette façon d'envisager l'ancrage des conditionnelles dans le discours antérieur est plus générale que celle dans laquelle on s'en tient aux seuls liens référentiels entre les participants à la prédication exprimée par la protase avec des participants déjà mentionnés. Elle présente surtout l'avantage de réintroduire dans la discussion le sens de l'adverbe (if/si, when/quand, ...) et de la subordonnée adverbiale qui guide la recherche du ou des contenu(s) précédemment évoqués à même de justifier le détachement en position préverbale.

\footnotetext{
${ }^{18}$ Cf. pour une présentation proche de Voguë (1999)
} 
Avec les adverbiaux non phrastiques (adverbes, SP, SN) détachés en tête de phrase, le problème se pose en des termes assez proches. Très souvent, leur occurrence est annoncée par une sorte de phrase amorce. C'est ce qui se passe par exemple dans :

(39) "Qu'est-ce que l'écriture? L'usage a consacré différentes acceptions du mot.

Dans une acception élargie, s'agissant de littérature et d'autres expressions artistiques, il peut désigner la manière de conduire un récit ou un exposé, au croisement des notions de "style» et de «forme». Ainsi parlera-t-on, de l'«écriture» de Jean-Luc Godard dans Pierrot le fou." (début d'article, Pour la Science)

où l'on retrouve, comme dans (35), un lien référentiel entre le SN régime du SP et un SN antérieur (en l'occurrence un lien partitif). Mais les relations de ce type sont, là aussi, loin d'être de règle. Dans l'extrait suivant, il est encore possible de dire que le SP réfère à un intervalle évoqué indirectement par la phrase immédiatement précédente qui "annonce" (mais après coup) une élaboration chronologique :

(40) "La stratégie de défense de la propriété intellectuelle choisie par Myriad est ainsi à comprendre comme un outil essentiel de construction de ce marché autonome des tests. Les rapports de la firme avec l'université de Pennsylvanie témoignent de cette dynamique. En 1998, suite à l'obtention de son premier brevet sur le gène BRCA1, Myriad Genetics adressa un " accord de collaboration 》 au service de génétique clinique de l'université de Pennsylvanie, alors principal laboratoire académique effectuant conjointement recherche des mutations BRCA, conseil génétique et suivi des personnes à risque.(La Recherche)

Le même raisonnement peut être appliqué à :

(41) "(...) Une véritable course aux mesures s'est engagée depuis quinze ans. Mais jusqu'ici, elles s'arrêtaient à 16 gigapascals. Seule une expérience d'ondes de chocs a produit les conditions requises, mais la vitesse obtenue reste difficile à interpréter.

Grâce à une nouvelle expérience menée à l'European Synchrotron Radiation Facility (ESRF) sur des échantillons comprimés en cellules à enclumes de diamant, Guillaume Fiquet de l'université de Paris-VI et ses collaborateurs accèdent à une mesure directe de la vitesse du son dans des agrégats cristallins de fer. (La Recherche)

où le $\mathrm{SP}$ avec un $\mathrm{SN}$ indéfini singulier sélectionne une expérience singulière sur un ensemble évoqué par l'idée de "courses aux mesures". Mais lorsque les SP figurent à l'initiale de texte (ce qui n'est pas rare) comme dans (42) et (43), il n'est plus possible de les rattacher à un segment du discours précédent :

(42) "En 1991, à la Station INRA de Dijon, Patrick Étiévant et Bruno Martin commençaient l'analyse du vin jaune, produit seulement dans le Jura. Le goût spécifique de ces vins résulte de leur technique d'élevage : on laisse le vin vieillir en tonneau pendant plusieurs années, sous un voile épais de levures Saccharomyces cerevisiae." (début d'un article sur le vin jaune, La Recherche)

(43) "Au XIXeme siècle, les romantiques comparaient la forêt au paradis terrestre. Certains discours écologistes actuels reprennent à leur compte cette idéalisation d'une forêt bénéfique: ils attribuent à la fois les fortes sécheresses et les inondations à sa disparition. La forêt a-t-elle des conséquences sur le cycle de l'eau? Certes, elle diminue l'écoulement annuel : I'évaporation et l'interception des précipitations par une forêt sont plus importantes que par les autres types de couvert végétal. (début d'un article sur la transpiration, La Recherche)

Les emplois de ce type sont relativement fréquents, notamment avec les SP adverbiaux spatiotemporels. Ce point est confirmé par Virtanen (1990) pour les adverbiaux temporels. Dans un corpus écrit composé de 84 textes de types différents (Survey of English Usage - 420000 mots), elle relève $1790 \mathrm{SP}$ ou SN adverbiaux temporels dont $34 \%$ figurent en tête de 
proposition et, sur ces 34\%, 60\% apparaissent en début de texte (les plus forts pourcentages se trouvant dans les journaux intimes, les lettres et les articles de presse).

Les deux études passées en revue dans cette partie portent sur les liens que peuvent entretenir les adverbiaux extraprédicatifs détachés en tête de phrase avec le contexte précédent. Les tendances relevées par les auteurs confirment globalement les analyses présentées dans la première partie à partir de l'examen d'exemples fabriqués. Même si, avec certains adverbiaux, l'antéposition apparaît comme la forme d'emploi non marquée, l'antéposition d'un complément non argumental est très souvent annoncée par le contexte ou rattachable après coup à celui-ci. Le fait que les adverbiaux (phrastiques ou non) détachés en position initiale s'appuient sur des informations déjà évoquées dans le discours antérieur explique qu'on les considère comme des topiques. Cette idée d'appui n'est cependant pas facile à préciser. On peut parler d'information "connue" ou "familière" mais ces notions demeurent assez imprécises. Partant de là, il est tentant de réduire cet appui à une forme de coréférence. Cette solution conduit cependant, et assez paradoxalement, à ignorer le rôle du constituant adverbial lui-même. Pour éviter ce biais, il semble préférable de partir de l'idée que les adverbiaux sélectionnent du fait de leur signification un attribut (temps, lieu, condition, cause, moyen, manière) des états de choses pour indexer la proposition en tête de laquelle ils apparaissent. Lorsque le critère sélectionné est générique, comme le temps et le lieu qui sont des traits inhérents à tous les états de choses, la décision consistant à l'exploiter comme un index n'a pas besoin d'être justifiée par le contexte précédent ce qui fait qu'ils peuvent apparaître à l'initiale de discours, surtout si le type de texte s'y prête. Lorsque le critère retenu pour indexer la proposition qui suit est moins prédictible, lorsqu'il met en avant un attribut particulier (comme le fait que telle action a été accomplie de telle manière, dans tel but, ...), on s'attend par contre à ce que son choix soit motivé par le contexte.

\section{III . LES ADVERBIAUX ANTEPOSES COMME TOPIQUES POUR L'AVAL}

Le fait que les adverbiaux antéposés puissent servir de topique pour la proposition en tête de laquelle ils sont détachés ne va pas de soi dans la mesure où celle-ci a en principe déjà un topique non marqué (généralement, son sujet). Rien n'empêche bien entendu qu'une phrase ait plusieurs topiques, cela arrive même très souvent (cf. Hasselgaard, à par. a), mais cela oblige à avoir une conception des topiques qui permet de spécifier aussi clairement que possible la fonction que chacun assume pour le contexte aval, proche mais aussi, comme nous allons y insister, lointain. Avant d'aborder ce dernier point nous allons présenter et discuter dans cette troisième partie une étude récente de Jacobs qui porte sur la relation topiquecommentaire et met essentiellement l'accent sur le lien que les expressions topicales entretiennent avec la proposition dans laquelle elles apparaissent.

\subsection{Les relations topique-commentaire selon Jacobs (2001)}

Jacobs développe une conception des relations topique-commentaire qui repose sur un ensemble d'attributs susceptibles d'être satisfaits conjointement ou seulement partiellement par certaines formes d'expression. Les formes satisfaisant l'ensemble de ces attributs sont des topiques "prototypiques" par rapport à celles n'en satisfaisant que certains.

Selon les langues, la relation topique-commentaire peut être codée, souligne Jacobs, lexicalement et/ou morphologiquement et/ou syntaxiquement et/ou phonétiquement. Les 
expressions remplissant une fonction de topique peuvent également être intégrées syntaxiquement (comme le sujet) ou non avec, dans ce cas, une différence entre les topiques disjoints qui sont liés référentiellement à un participant à la prédication et certains adverbiaux qui ne le sont pas (cf. ci-après). Les constructions qui ont été décrites comme tombant sous le coup de la distinction topique-commentaire au sein d'une même langue sont également diverses. C'est le cas notamment en allemand où, dit Jacobs, il est possible de faire valoir que la relation sujet-verbe dans les phrases catégoriques (/PEter ISCHLÄFT - Pierre dort), la dislocation à gauche (/PEter der kommt IMORgen - Pierre, il viendra demain), les "hanging topics" (|Peter, ich habe ihn heute nicht ge।TROFfen - Pierre, je n'ai lui hier pas rencontré), les "free topics" (Was Peters Ge/BURTStag betrifft, so habe ich - En ce qui concerne l'anniversaire de Pierre, je ne sais pas quel cadeau lui faire), et les constructions avec "Itopicalisation" ( |/JEden Freund Peter kenne ich $\mid$ NICHT-Chaque ami de Pierre connaître je $n e g)$ sont des topiques. Ces emplois ne présentent aucun trait sémantique et pragmatique justifiant qu'on les range dans une même catégorie fonctionnelle, toutefois:

"what is behind the intuition of linguists that all these constructions are instances of Topic-Comment is the fact that they all sufficiently ressemble prototypical examples of Topic-Comment. With these prototypical cases they share some salient semantic attributes, but not necessarily the same semantic attributes and not necessarily all the relevant attributes. (...) There is no unitary functional notion underlying all TopicComment constructions in natural languages. Rather, in regard to their semantic and pragmatic properties these constructions are related only by similarities to prototypical examples" (642-643)

Pour Jacobs, les notions de topique et de commentaire ne peuvent être définies qu'en termes d'air de famille, comme les parties du discours, les relations grammaticales, ou les valences. Elles ne reposent pas sur des attributs nécessaires et suffisants mais jouent sur "quatre attributs sémantiques saillants" ou "dimensions de la relation Topique-Commentaire", à savoir : la séparation de l'information, la prédication, l'adressage ("addressation") et le cadrage ("frame-setting").

Le trait "séparation de l'information" prévoit que le topique est séparé informationnellement du commentaire : "In (X Y), X is informationally separated from Y iff the semantic processing of utterrances of (X Y) involves two steps, one for X and one for $\mathrm{Y}^{\prime \prime}$ (p. 645). La séparation se traduit au niveau de l'intonation mais elle influence aussi certains mouvements syntaxiques ${ }^{19}$. Un constituant $\mathrm{X}$ est intégré à $\mathrm{Y}$, quand $\mathrm{X}$ est un argument syntaxique de $\mathrm{Y}$, quand $\mathrm{Y}$ assigne un theta-rôle à $\mathrm{X}$, quand $\mathrm{Y}$ n'assigne pas une "une propriété spatio-temporelle illimitée à $\mathrm{X}$ " (scénique) et quand "X ne contient pas plus d'un argument lexical" (opposé à "fonctionnel"). Dans les autres cas, $\mathrm{X}$ est non intégré. La séparation est un trait caractéristique des adverbiaux (Mit /SICHHerbeit wird Peter IZUstimmen. Avec certitude, va Pierre accepter), mais elle n'est pas le seul critère à prendre en compte pour statuer sur leur intégration : les adverbes modaux de probabilité (comme avec certitude) ne sont normalement pas considérés comme des topiques, même quand ils sont détachés à gauche.

La prédication permet de différencier les attributs de la relation topique-commentaire qui sont présents dans les phrases catégoriques mais pas dans les constructions avec un adverbial modal. Elle passe par la définition de ce que Jacobs appelle le sujet sémantique : "In (X Y), $\mathrm{X}$ is the semantic subject and $\mathrm{Y}$ the semantic predicate iff (a) $\mathrm{X}$ specifies a variable in the semantic valency of an element in $\mathrm{Y}$, and (b) there is no $\mathrm{Z}$ such that (i) $\mathrm{Z}$ specifies a

\footnotetext{
${ }^{19}$ Par exemple, en français, seuls les éléments intégrés peuvent être extraposés et focalisés.
} 
variable in the semantic valency of an element in $\mathrm{Y}$ and (ii) $\mathrm{Z}$ is hierarchically higher in semantic form than $\mathrm{Y}^{\prime \prime}$ (p. 647). Dans les phrases catégoriques du type : / PEter $\mid$ SCHLÄFTPeter dort, le verbe introduit une variable du fait de sa valence, variable que le nom propre spécifie : Peter est le sujet sémantique du verbe principal (condition bi). Dans les constructions avec adverbe modal antéposé, l'adverbe ne spécifie pas une variable qui serait introduite par un autre constituant de la phrase, cette variable s'applique à la phrase entière, et l'adverbe n'est pas sujet sémantique. Les adverbiaux spatiaux antéposés (In der /KÜCHe hat Peter GelSCHIRR gespült - Dans la cuisine, Pierre a fait la vaisselle) saturent par contre une variable de la situation qui est inscrite dans la sémantique du verbe de sorte que, occupant la position sémantique la plus élevée, ils sont sujets sémantiques de la phrase. Cette prédiction est confirmée par le fait que de nombreuses langues, comme le hongrois, traitent les adverbiaux spatio-temporels comme des topiques.

L'adressage est destiné à capter le fait que le commentaire est à propos du topique ce dont ni la séparation, ni la prédication ne rendent compte. L'adressage se définit comme suit : "In (X Y), X is the address for Y iff X marks the point in the speaker-hearer knowledge where the information carried by $\mathrm{Y}$ has to be stored at the moment of the uttrerance of (X Y)". Lorsque l'auditeur-lecteur interprète une proposition, il a à l'esprit, dit Jacobs, un ensemble de propositions qui sont connues. Ce "context-set" change constamment au fur et à mesure de l'avancée dans le texte. L'organisation thématique facilite le stockage des informations nouvelles en les rapportant à un sous-ensemble limité de ce context-set. Les propositions rassemblées sous une même adresse constituent des sortes de fichiers et on s'attend à ce que

"the propositions in the speaker-hearer knowledge at a given moment in the discourse are tied up in bundles that can be compared to files on different subjects, namely those subjects the propositions pertain to (...) Adress is a constituent that - via its reference identifies one of these mental files : it refers to the entity that is the subject of the file. The complementary part of the sentence corresponds to a proposition that has to be entered into the file identified by the address. (...) Data structures of this type kind occur outside of language, for example in archives and catalogues" (p.651).

Reinhart (1981) qui reprend la notion de "context-set" à Stalnaker (1978) fait le rapprochement avec les fichiers dans la documentation, mais elle suggère que cette fonction est une caractéristique essentielle des topiques, alors que, pour Jacobs, il ne s'agit qu'un des quatre traits prévus dans sa conception prototypique. A propos du catalogage des documents, Jacobs fait remarquer qu'il y a des traits qui ne font jamais l'objet d'une indexation. Par exemple, on ne trouvera jamais dans un fichier de fiches intitulées "un (quelconque) auteur allemand du XIXème siècle" ou "chaque auteur allemand du XIXème siècle" ou "aucun auteur allemand du XIXème siècle". Pour qu'un constituant puisse assumer une fonction d'adressage, même s'il est sujet sémantique, il faut qu'il réfère à une entité spécifique. La preuve en est qu'en allemand et en hongrois il n'est pas possible d'avoir un indéfini en position de free-topic. Mais Jacobs reconnaît qu'il y a des problèmes, notamment avec le japonais, où $w a$, bien que marquant un topique, est possible avec un SN indéfini quantifié. Les adverbiaux thématisateurs (du type de à propos de/concernant $X$ ) avec free topic (sans reprise) adressent un fichier, mais ils ne spécifient pas une variable actancielle du verbe, ce qui fait qu'il y a adressage sans prédication. Pour qu'un constituant remplisse une fonction d'adressage, il faut qu'il réfère à une entité spécifique et identifiable, comme le prévoit Lambrecht (1994). Cette condition ne vaut cependant pas pour les topiques en général. Elle n'intervient que quand on examine le rapport topique-commentaire dans la dimension particulière que constitue l'adressage (la fonction de stockage de l'information dans un fichier). Il en va probablement de même pour la définitude et la saillance et avec les traits "familiers" ou "présupposés". Mais il n'est pas clair, dit Jacobs, que ces caractéristiques, contrairement à la spécificité- 
identifiabilité, soient nécessaires, il se pourrait qu'ils n'apparaissent que dans certaines constructions.

L'adressage n'est pas un trait que satisfont toutes les constructions avec topique. Une phrase comme (je traduis) : Physiquement, Paul va bien, ne dit pas quelque chose à propos de "physiquement". De même, une conditionnelle comme Si l'équipe gagne, ils recevront une commande de la Présidence n'est pas énoncée à propos de si l'équipe gagne. Les adverbiaux de ce type remplissent une autre fonction, une fonction de cadrage qui consiste à "restreindre l'application de la proposition exprimée par le reste de la phrase à un certain domaine" (p.656). Ces constructions ont été considérées comme des instances de la relation topiquecommentaire par de nombreux auteurs comme Chafe (1976) qui relève (p.51) que "real topics' (in topic prominent languages) are not so much 'what the sentence is about' as 'the frame within which the sentence holds" (voir également : Haiman et Ducrot cités dans la partie précédente). Partant, Jacobs définit le cadrage comme suit : "In (X Y), X is the frame for Y iff $\mathrm{X}$ specifies a domain of (possible) reality to which the proposition expressed by $\mathrm{Y}$ is restricted" (p.656).

L'adressage n'a aucune fonction dans la validation de la proposition. Il consiste seulement à envoyer une information dans un fichier déterminé, pas à limiter les conditions dans lesquelles celle-ci est avérée. Ces deux fonctions sont différentes mais "they both introduce a background against which the information conveyed by an utterance has to be interpreted, thereby guiding semantic processing in certain direction" (p.656-57). Dans Physiquement, Pierre va bien, Jacobs considère que l'adverbe de domaine sature une variable de la situation :

[PHYSIQUEMENT(y) \& [PIERRE $\left.\left.(x) \& \operatorname{ETRE-BIEN~}\left(x, y_{\text {dom }}\right)\right]\right]$

ce qui fait qu'il est aussi le sujet sémantique. Dans ce cas, il y a, dit-il, coïncidence entre le sujet sémantique et le cadre (les traits prédication et cadrage sont satisfaits). Par contre, avec: Dans mon rêve, Pierre était un crocodile, on n'a pas affaire à la même structure :

Dans mon rêve ([Pierre $(x)$ \& était-un-CROCODILE $(x)]$ )

Le SP n'introduit pas une variable et n'est pas un sujet sémantique, c'est un foncteur qui porte sur l'ensemble de la proposition et qui quantifie sur les mondes possibles. Mais, note Jacobs, certains auteurs (il cite Maienborn et Klein) introduisent une variable temporelle, qui représente la situation pour laquelle la phrase entière est assertée :

\section{[DANS MON REVE(S) \& IL-SE-TROUVE-QUE ([PIERRE $(x)$ \& ETAIT-UN- CROCODILE $(x)] \mathrm{s}$}

$\mathrm{Si}$ on suit cette analyse, le SP est à la fois cadratif et prédicatif parce qu'il spécifie une variable événementielle attachée au (méta-)prédicat IL SE TROUVE QUE. Jacobs relève que les conditionnelles antéposées pourraient également être analysées de ces deux façons : une dans laquelle elles ne sont pas prédicatives mais cadratives, l'autre dans laquelle elles sont à la fois prédicatives et cadratives, sachant qu'elles satisfont par ailleurs au trait séparation mais pas au trait adressage.

\subsection{Discussion}

L'article de Jacobs mériterait une présentation plus approfondie mais les éléments rapportés ci-dessus devraient suffire pour la discussion des points qui suivent et de ceux que nous aborderons dans la partie suivante.

Le trait "séparation" ne fait pas problème avec les adverbiaux antéposés qui sont compléments de phrase et extraprédicatifs. Il permet, en français, de différencier les 
adverbiaux cadratifs des modaux qui sont difficilement détachables en position préverbale et se prêtent à des emplois prédicatifs (forcément / probablement / peut-être que, il est nécessaire / possible / certain que) exclus avec les cadratifs comme physiquement / statisquement / honnêtement... Les adverbes modaux du type de forcément, nécessairement, probablement, peut-être, etc. sont des opérateurs vériconditionnels qui affectent le calcul de la vérité/fausseté du contenu de la proposition dans laquelle ils figurent. Par contre, les adverbiaux cadratifs sont clairement séparés et cette séparation suffit, comme nous l'avons souligné dans la première partie, pour qu'ils interviennent, non pas sur le contenu propositionnel de la proposition en tête de laquelle ils apparaissent, mais sur le fait qu'elle dénote, ce qui n'est possible qu'une fois que celui-ci a été construit comme tel, donc une fois que sa valeur modale a été calculée (Charolles 1997).

Concernant la prédication, Jacobs considère, dans l'esprit de la notation davidsonnienne, que les adverbiaux spatiaux et temporels détachés en tête de phrase saturent une variable de la situation et sont des sujets sémantiques dans la forme sémantique sousjacente où ils occupent une position dominante. Cette décision, qui peut se justifier par le fait que l'espace et le temps sont des dimensions génériques des états de choses, tend cependant à en faire des adverbiaux intraprédicatifs et elle ne permet pas de les différencier des emplois où la séparation a lieu en final et où elle force le rattachement à la prédication (cf. I. ci-avant). Elle ne permet pas non plus d'expliquer pourquoi les compléments de lieu antéposés prennent facilement une valeur temporelle, ce qui n'est pas le cas quand ils sont posposés. Pour expliquer cette différence, il faudrait faire intervenir le fait que les compléments postposés ne sont pas "dominants" dans la forme sémantique, sauf que cette relation de dominance n'est pas clairement définie par Jacobs et qu'on ne voit pas bien comment elle pourrait expliquer ces effets.

Jacobs considère que les adverbes comme physiquement saturent une "variable de domaine". Par contre, pour les conditionnelles et les adverbiaux créateurs de monde (dans mon rêve), il présente une analyse dans laquelle ceux-ci ne sont pas des sujets sémantiques tout en reconnaissant qu'il serait possible de les rattacher à un prédicat événementiel sousjacent (il se trouve que) et donc de leur conférer ce statut. Cette différence de traitement n'est pas justifiée en détail, mais les analyses développées par l'auteur tendent plutôt, dans leur ensemble, à faire autant que possible des adverbiaux antéposés des sujets sémantiques. Cette tendance, outre qu'elle conduit aux difficultés que l'on vient de relever à propos des adverbiaux spatiaux, paraît difficilement tenable avec un certain nombre de circonstants. Que faire en effet avec les compléments de cause (à cause de $X$ ), d'opposition (malgré X, en dépit de $X$ ), de condition (sans $X$, avec $X$ ), de moyen (grâce à $X$, à l'aide de $X$ ) ? Que faire avec les infinitives de but (pour montrer que $X$ ) ? avec les participiales ou les constructions absolues descriptives (Avec le sourire/le sourire aux lèvres, $X$ ) qui, quoique comportant un sujet vide coréférentiel avec un constituant de la proposition qui suit (en général avec le sujet), ne sont pas adressées (la proposition ne portant pas sur elles) ? Plus on s'écarte des compléments potentiellement vériconditionnels (ce que les adverbiaux antéposés ne sont de toute façon pas, cf. ci-après), plus la décision de les rapporter à un prédicat passe partout sous-jacent dont ils instancieraient une variable risque de paraitre ad hoc. On voit encore plus mal comment il serait possible de dire que, dans les phrases préfixées par des SP du genre de par hasard, par dépit, par chance, par bonheur qui ne sont même pas référentiels, ceux-ci pourraient être des sujets sémantiques. Pire encore avec les adverbiaux organisateurs (d'une part/d'autre part, d'un côtélde l'autre, enfin), "énonciaitfs" (franchement, justement) et conjonctifs (justement, mais, sinon, autrement,...), qu'il n'y a aucune raison d'écarter de la discussion. 
Concernant l'adressage, dont la forme prototypique est la dislocation avec reprise pronominale, Jacobs différencie les emplois comme In der /KÜCHe hat Peter GelSCHIRR gespült (Dans la cuisine, Pierre a fait la vaisselle) dans lesquels il y a +Séparation, +Prédication, -Adressage et +Cadrage, des emplois comme In der /KÜCHe, da hat Peter GelSCHIRR gespült où il y a adressage du fait de la présence de da (là). Les emplois de ce type ne sont pas exclus en français (Sur le pont d'Avignon, on y danse, cf. Prévost dans ce volume) mais ils semblent assez rares (au moins à l'écrit), et sans équivalent du coté temporel. Avec les SP spatiaux repris (In der /KÜCHe, da hat Peter GelSCHIRR gespült), Jacobs note que ce qui est adressé en mémoire, ce n'est pas le seul référent du SN régime, mais la région de l'espace dénotée par le SP (ce qui l'oblige à rajouter une variable référentielle "r" à la variable "s" déjà explicitée pour marquer que le SP est un sujet sémantique). Toutefois, avec les médiatifs (selon X, d'après X), la reprise de X qui n'est pas exclue (Selon Chomsky, il...) implique des contextes particuliers et ne met pas en cause le SP entier comme c'est le cas avec les locatifs, à moins de dire, ce que ferait peut-être Jacobs, que selon $X$ identifie un site abstrait, ce qui ne va pas de soi. En français, les topicalisateurs (quant $\grave{a} X$, concernant $X$, ̀̀ propos de $X$, etc.) impliquent (en principe) la reprise (cf. Combettes dans ce volume) et donc l'adressage. Ce fait est révélateur : la reprise signale que la proposition porte sur le référent du $\mathrm{SN}$, comme dans les dislocations du sujet où il a y a séparation de la référence et du rôle (Lambrecht 1994), l'adverbial se "contentant" de préciser que le référent doit être sélectionné parmi un ensemble de candidats accessibles en amont, de même que, avec les médiatifs, il indique une façon d'accéder au référent du SN régime (cf. Schrepfer à par.) ${ }^{20}$.

Telle que la présente Jacobs, l'opposition entre adressage et cadrage joue sur la référence : seuls les contenus propositionnels portant sur un référent (spécifique, etc. ) sont adressés. Le commentaire est à propos des expressions exprimant ces référents et il est stocké dans un fichier qu'elles indexent. A l'inverse, les expressions purement cadratives spécifient, dit Jacobs, le domaine (possible) de la réalité dans lequel la proposition est valide. Cette fonction (véridictionnelle et non vériconditionnelle) convient parfaitement pour si, pour les prédicats créateurs de monde ( $X$ rêver/se figurer que), pour les localisateurs concrets et abstraits comme les SP praxéologiques du genre de en biologie (Vigier, à par.), bref pour toutes les expressions à même de restreindre l'univers du discours qu'elles soient sujets sémantiques ou non, adressées ou non. Si l'on s'en tient à la définition de Jacobs seuls les adverbiaux de ce type devraient être "frame-setting". Cette position suppose qu'il est possible de différencier, parmi les circonstances associées à un état de choses, celles qui conditionnent son existence et celles qui n'ont pas ce pouvoir. Or rien n'est moins sûr. Que faire, encore une fois, avec tous les compléments adverbiaux antéposés qui ne sont ni sujets logiques ni adressées et qui font allusion à une "circonstance" ne spécifiant pas le domaine de la réalité dans lequel la proposition exprimée est valide, comme les compléments de manière, de but, etc. ? Se contenter de dire qu'elles satisfont au trait +Séparation paraît un peu court, ne seraitce que parce que ces expressions, comme celles limitant l'univers du discours, sont à même d'indexer plusieurs propositions. Jacobs n'envisage pas cette possibilité, non plus d'ailleurs que Ramsay et Ford \& Thompson dont nous avons présenté les analyses dans la partie précédente $^{21}$. Ce point, comme nous allons le montrer dans la partie suivante, n'est pourtant pas sans conséquence.

\footnotetext{
${ }^{20}$ Jacobs note qu'en allemand le reprise d'un complément de manière à l'aide de so est souvent problématique, comme leur équivalent en français : *Grâce à Paul, ainsi tu pourras le rencontrer.

${ }^{21}$ Ramsay parle de "portée", mais en amont et dans un sens particulier puisqu'il s'agit de la distance séparant la subordonnée (et singulièrement des expressions référentielles qu'elle comporte) avec une phrase l'annonçant dans le texte précédent. Quant à Ford \& Thompson, elles soulignent en conclusion que les "subsequent propositions
} 


\section{LES ADVERBIAUX CADRATIFS ET LES TOPIQUES DE DISCOURS}

Dans une étude consacrée aux infinitives de but, Thompson (1985) montre que quand celles-ci sont antéposées, elles sont à même de porter sur plusieurs propositions apparaissant dans la suite, pouvoir qu'elles n'ont pas quand elles sont postposées et où elles se trouvent rattachées à la prédication. Elle relève, en se fondant sur le même corpus que Ford \& Thompson (ci-avant), que cette portée s'étend en moyenne sur 3,18 propositions et ajoute que le "poids" en nombre de mots des propositions principales est beaucoup plus important quand elles apparaissent à la suite d'une infinitive détachée en tête que quand cette subordonnée est en position finale (sur 18 propositions finales et initiales prises en compte, la principale qui suit une infinitive de but compte en moyenne 17,5 mots et seulement 7,8 quand elle la précède).

Ces indications sont intéressantes mais Thompson s'en tient là. Elle ne précise pas en particulier sur quels indices elle s'est appuyée pour décider que la portée d'un infinitive de but antéposée devait être étendue ou non à une ou n phrases faisant suite à celle dans laquelle elle figure. Charolles \& Lamiroy (2002) traite de cette question à partir d'exemples comme :

(44) "Le recrutement est loin d'être uniforme sur l'ensemble du territoire. Pour le mettre en évidence, nous avons pris en compte, pour la fin des années 1980, les étudiants admis aux concours, qui représentent l'essentiel des "nouveaux recrutés" par académie d'inscription. Ce nombre d'étudiants recrutés a été rapporté à celui des premières affectations par académie, c'est-à-dire au nombre d'enseignants débutants titularisés, qui exprime grossièrement les besoins en professeurs. Les disparités sont considérables entre les deux extrémités du gradient nord-sud : au nord de Paris, le recrutement d'étudiants locaux couvre moins du tiers des besoins, alors qu'il y a deux à quatre fois plus d'admis que de premiers postes dans les académies les plus méridionales. " (Atlas de la France scolaire)

Nous montrons, en nous fondant sur des arguments sémantiques mais aussi syntaxiques, que les infinitives de but antéposées ne peuvent indexer que les propositions faisant référence aux moyens mis en oeuvre par les participants prenant en charge le but exprimé dans les infinitives, mais pas les résultats qu'ils obtiennent à la suite de cette mise en oeuvre. Ce point, que nous détaillons, est tout à fait sensible dans l'extrait ci-dessus où l'infinitive porte sur tout le passage en caractères droits, mais pas sur l'exposé des données recueillies, quoique cet exposé soit attendu. Les propositions tombant sous la portée de l'infinitive se différencient de la présentation des résultats par le changement de temps verbal et par le fait qu'elles mettent en jeu le pronom sujet de la première phrase, pronom qui lie le sujet vide de l'infinitif et que l'on retrouve dans le passif a été rapporté à. À cela, on ajoutera que la reprise par le SN démonstratif ce nombre d'étudiants d'un SN précédemment introduit favorise l'extension de la portée de l'infinitive.

Dans (44) et les autres exemples que nous examinons dans Charolles \& Lamiroy, les infinitives délimitent des segments de discours homogènes relativement au trait spécifié par l'adverbial qui les initie, en l'occurrence le but poursuivi par les instigateurs du procès. Ces segments, qui peuvent se réduire à la seule proposition hôte de l'adverbial, constituent des cadres de discours (Charolles 1997) à la fois parce qu'ils sont introduits par un adverbial

take the content of the if-clause as their necessary background." ( p.370) sans toutefois examiner ce qui se passe au-delà de la principale. 
cadratif et parce qu'ils constituent des blocs au sein du texte. Cette conception des cadres rejoint celle de Jacobs sur le premier point, mais elle s'en différencie sur plusieurs autres.

L'approche adoptée accorde une atttention particulière au contenu sémantique du critère exploité par l'adverbial (aspect déjà souligné en 2.2) mais aussi au contenu de la et des propositions qu'il indexe. Quand on se limite au seul commentaire constitué par la proposition en tête de laquelle apparaît l'adverbial, certains aspects sémantiques de cette relation risquent de passer inaperçus dans la mesure où le placement force à établir un lien avec le commentaire. Ces aspects ne se révèlent pleinement que quand on prend en considération la suite du discours, où certains indices, comme le temps verbal dans (43) et les emplois apparentés, jouent un rôle déterminant pour la continuation du cadre.

La place peut d'ailleurs suffire pour induire de tels liens, la présence d'un adverbial n'étant même pas nécessaire pour qu'il y ait cadrage. A l'écrit, notamment dans les textes scientifiques et techniques, on trouve en effet des constructions comme:

(45) "A ces questions on se doute qu'il n'y a pas de réponses simples. D'après les données présentées en figure 2, ni la richesse ni les dépenses de santé mobilisées par l'Etat ne peuvent expliquer les différences dans l'évolution des espérances de vie. (...)

Richesse et santé. L'absence de corrélation entre les dépenses de santé et l'espérance de vie dans les pays de l'OCDE est une notion relativement peu intuitive. Pendant longtemps, on a cru qu'il suffisait de rendre accessibles de façon universelle les services médicaux, l'accès à l'hôpital, et surtout les médicaments, pour que la santé de la population s'améliore et que les disparités entre les différents groupes sociaux s'effacent. Or, aujourd'hui, il faut reconnaître que cet objectif n'a pas été atteint : les disparités face à la santé se sont conservées malgré l'apparition de la médecine dite " scientifique ".

En réalité, la santé, mesurée par l'espérance de vie, est plus fortement influencée par l'ensemble de l'organisation sociale (conditions de vie et position sociale des individus) que par des politiques spécifiques de santé ou par tel ou tel système de soins. "." (La Recherche)

où les $\mathrm{SN}$ non déterminés détachés en tête de paragraphe fonctionnent comme des sortes de topiques, dont le paragraphe entier est le commentaire. Ces topiques, généralement annoncés par une phrase introductrice, se confondent avec les topiques de discours. Ils mettent en exergue une ou des information(s) que le rédacteur considère comme utile(s) pour guider la lecture du passage. Leur lien avec les informations qu'ils indexent est purement sémantique, mais, point remarquable, leur portée est marquée par l'alinéa.

Si les adverbiaux fixent un critère pour l'interprétation de la proposition en tête de laquelle ils sont détachés, si ce critère sert, comme dit Chafe (1984: 447-448) de "guidepost" pour l'interprétation de celle-ci, si donc ils assument une fonction procédurale, on ne voit pas quel argument imposerait que cette fonction se réduise à cette seule proposition. Cette dimension cognitive est minorée dans l'étude de Jacobs, mais elle ressort plus ou moins dans la distinction qu'il établit entre adressage et cadrage. L'idée selon laquelle seuls les topiques adressés donneraient lieu à l'ouverture de fichiers dans lesquels seraient rassemblées les informations "à propos" des référents associés à ces topiques, joue en réalité sur deux dimensions qui, comme nous voudrions le montrer, ne se recoupent pas.

Pour ce qui est de l'ouverture d'un fichier et du stockage d'informations, Jacobs s'en tient à une présentation désincarnée des opérations que ce stockage peut impliquer. Peut-être 
a-t-il en tête une mémoire artificielle associée à un analyseur automatique, mais il n'y a aucune raison de s'en tenir à ce point de vue. Le traitement de (44) et (45) implique de facto que les lecteurs gardent en mémoire les indications attachées aux expressions graissées dans le texte pour le traitement des passages (en caractères droits) qu'ils indexent. Si l'on aborde la question sous cet angle, tous les adverbiaux antéposés adressent un critère destiné à indexer un ou plusieurs commentaires.

Pour ce qui est maintenant de leur à-propos (aboutness), la reprise du référent de l'adverbial qui est, pour Jacobs, une condition nécessaire de l'adressage, joue effectivement un rôle, mais le problème est plus général. Imaginons le début de texte suivant:

(46a) Lola sortit faire un tour. En bas de l'immeuble, elle croisa le facteur.

(46b) Lola sortit faire un tour. En bas de l'immeuble, un homme faisait les cents pas.

Dans les deux cas, le fait dénoté par la seconde proposition réfère à ce qui se passe au bas de l'immeuble et, on peut dire, si l'on veut, que le SP instancie une variable de la situation dénotée par la prédication. Cette variable n'est pas reprise dans la seconde proposition par un pronom comme là, mais cela n'est pas crucial pour savoir si la seconde phrase est ou non à propos de en bas de l'immeuble. On sent bien, intuitivement, qu'il y a déjà une différence entre (46a) et (46b) quoiqu'il n'y ait reprise ni dans (46a) ni dans (46b). Cette différence ressort clairement quand on complète les deux exemples de la façon suivante:

(46a') Lola sortit faire un tour. En bas de l'immeuble, elle croisa le facteur. Il portait un énorme sac et elle lui proposa de l'aider.

(46b') Lola sortit faire un tour. En bas de l'immeuble, un homme faisait les cents pas. Une fine couche de givre recouvrait le sol. Des enfants rentraient de l'école en se chamaillant.

Dans (46a'), le circonstant spatial se contente de fixer le cadre où se déroule une série d'événéments qui ont une cohérence indépendamment de l'endroit où ils se produisent. Ce cadre sert d'arrière-plan, il n'est pas le topique adressé du commentaire ni des phrases qui suivent, ce qui ne l'empêche pas, il faut le souligner, de porter sur les deux phrases faisant suite à la proposition hôte du SP. Dans (46b'), le texte est par contre à propos de ce qui se passe dans le lieu indiqué par le SP antéposé, les événéments n'ont d'autre point commun que de se dérouler dans le même endroit et cela suffit pour conférer au passage une cohérence a minima $^{22}$. Le SP indexe, comme dans (46a'), l'ensemble de la séquence et les propositions sont comprises comme des comptes rendus de perception affectés à Lola. Les marques aspecto-temporelles et les types de procès jouent dans la discussion de ces exemples un rôle aussi important que les phénomènes de reprise anaphorique. Ces marques, présentes dans (46a) et (46b), favorisent d'emblée les interprétations confirmées dans leur version étendue. Il y a cependant une différence entre les deux exemples, puisque (46b) pourrait donner lieu à une version comme :

(46b") Lola sortit faire un tour. En bas de l'immeuble, un homme faisait les cents pas. Il l'aborda pour lui demander si les taxis étaient en grève.

où le SP serait cadratif, alors qu'il est plus difficile, avec un début comme (46a), d'imaginer une suite qui serait à propos de ce qui se passe en bas de l'immeuble et non de Lola ou du facteur.

P.Le Goffic (1994) discute des exemples comparables. Après avoir noté que les circonstants initiaux donnent "le cadre de la phrase", il relève que celui-ci "peut constituer un véritable thème ou une partie du thème (en liaison avec le sujet).": "même s'il n'apparaît pas, psychologiquement, comme ce dont l'énoncé parle, le circonstant initial de temps ou de lieu

\footnotetext{
${ }^{22}$ Cf. Charolles (1995)
} 
sert de repère, permettant de localiser ou de dater un événement ou une situation dans son ensemble" (p: 464). Il note à ce sujet que, dans un énoncé comme :

(47) La nuit, tous les chats sont gris.

"il n'est pas évident de dire quel est le thème : est-ce le sujet les chats ? est-ce le circonstant la nuit (en comprenant que la phrase vise à caractériser la nuit et non les félins ? est-ce l'association du sujet et du circonstant ("orienté-sujet") "les chats-la nuit" ? (p: 15). Le choix d'une interprétation ou d'une autre dépend, à notre avis, de la suite. Si on enchaîne sur : tous les chiens sont des loups, tous les hommes sont des ennemis, etc., le lecteur ou l'auditeur comprendront rétrospectivement que le thème de la première phrase était "ce qui arrive la nuit". Si au contraire la suite est du type : ils rôdent lamentablement autour des maisons, ils hurlent sous les porches, etc., ils comprendront que le thème est les chats ou, plus exactement, comme dit P.Le Goffic, les chats-la nuit, (i.e. : leur comportement nocturne) dans la mesure où ces propositions sont indexées par le SP temporel. Le fait que (47) soit un proverbe incite en faveur de la première lecture, dans un sens au demeurant plus large : le thème n'étant pas, à proprement parler, ce qui arrive la nuit, mais ce qui se passe dans toutes les situations confuses.

Si les analyses qui précèdent sont fondées, elles montrent qu'il n'est pas possible de statuer sur l'à propos (et donc l'adressage) des circonstants antéposés à partir du seul contenu de la proposition les accueillant ${ }^{23}$. Il faut tenir compte du topique du discours, lequel varie au fur et à mesure que celui-ci avance. La plupart du temps, les circonstants détachés en tête de phrase se contentent d'encadrer leur proposition d'accueil et une ou plusieurs autres apparaissant ensuite. C'est ce qui arrive dans l'extrait suivant :

(48) (...) les études sur l'intonation ne datent pas d'hier. Déjà dans les années 1930, les linguistes du groupe de Prague avaient conçu l'intonation dans une perspective moderne ; et nos amis britanniques, confrontés à de difficiles problèmes de pédagogie dans l'enseignement de la langue anglaise avaient devancé leurs collègues du continent et $d u$ monde dans la recherche des formes prosodiques. Pourtant, au Sixième Congrès des Sciences phonétiques à Prague en 1967, sept communications seulement étaient consacrées à l'intonation. Aussi, ... (Rossi (1999) avant-propos)

où les circonstants graissés ne font que baliser les étapes du développement des études sur l'intonation dans la linguistique contemporaine, sujet qui est au centre du propos de l'auteur. Comme ce propos est historique, les compléments de temps sont liés au topique du passage. Rien de plus normal, donc, qu'ils servent, en coulisse, à répartir des connaissances communiquées. Leur usage en série est typique dans ce genre de contexte. On le voit bien dans (48) mais aussi dans l'extrait suivant :

(49)"L'écriture est attestée très anciennement, et dans des civilisations fort éloignées dans l'espace. Chronologiquement, c'est en Mésopotamie, vers 3300 avant notre ère, qu'apparaissent les premières écritures, auxquelles il faut probablement associer d'autres écritures plus à l'Est, en Élam. Peu après, voici les premiers témoignages indiscutables d'une écriture égyptienne (vers -3200). Puis, vers 2000 avant notre ère, une autre écriture est connue dans la vallée de l'Indus, et, vers 1500 avant notre ère, se manifeste pour la première fois l'écriture chinoise. Encore plus tard, naissent les écritures méso-américaines, chez les Mayas et les Olmèques. Les foyers sont très dispersés et indépendants. Au plus, on peut hasarder que l'idée même d'écriture a pu passer de Mésopotamie en Égypte, mais, par ailleurs, il n'y a assurément aucun lien génétique entre ces différentes écritures, malgré quelques

\footnotetext{
${ }^{23}$ Pour une discussion cf. Terran (2001) et Prévost (à par.)
} 
théories peu convaincantes pour en établir un." (Pour la science)

Pour communiquer les informations dont il fait état dans cet extrait, l'auteur de (49) pouvait choisir de les distribuer dans des cadres temporels ou spatiaux, ces deux critères étant également annoncés dans la première phrase. Or on voit bien qu'au lieu d'exploiter tantôt l'un et tantôt l'autre, le rédacteur réserve cette fonction aux seuls adverbiaux temporels, les indications de lieu étant reléguées en fin de phrase (adverbiaux non détachés) ou exprimées par des adjectifs. Ce mode d'organisation, qui exploite au maximum le potentiel cadratif et contrastif des adverbiaux antéposés, est très courant. Cette exploitation contrôlée suppose plus ou moins une planification à l'avance avec, probablement, des retouches en cours de route. On peut parler, comme Enkvist (1987), Virtanen (1990) et Hasselgaard (à par. b) de stratégie, en insistant toutefois, comme le font d'ailleurs ces auteurs, sur le fait que ce mode d'organisation cadrative n'est en général exploité que sur des fragments qui s'y prêtent comme dans (49). Rares sont les textes entièrement structurés de cette façon ${ }^{24}$.

Il est plus difficile de trouver des textes organisés de la même façon mais à partir de SP adressés et donc topiques dans le sens d'à propos. Dans les narrations, certaines séquences descriptives prises en charge par un sujet percevant peuvent être structurées de cette manière, mais ces séquences restent à propos de ce que perçoit un personnage. Les cas comme (50) qui est extrait d'un programme TV :

(50) "En Allemagne, les choses vont de mal en pis pour Helen. Kurt lui enlève son fils Johannes. Jack découvre que Caroline continue à prendre de l'hérö̈ne. Au Pakistan, Roquia essaie d'arrêter un important trafiquant. Mais celui-ci verse des pots de vin au juge ..." (présentation du film Traffik-Le sang du pavot)

sont assez exceptionnels. Dans (50), les SP de lieu délimitent deux cadres regroupant l'ensemble des propositions du texte. La seconde proposition élabore celle en tête de laquelle apparaît le premier SP. Par contre, le fait que Jack découvre que Caroline continue à prendre de l'héroïne n'entretient aucun rapport avec ce qui précède, si ce n'est que l'on comprend que cette découverte doit avoir lieu quelque part en Allemagne, ce qui montre que les SP antéposés jouissent d'un potentiel cadratif qui tend, sauf indication contraire, à s'exercer audelà de leur phrase hôte. Ce pouvoir est limité dans (50) par l'occurrence d'un autre SP dont la portée s'étend à la dernière phrase du texte qui est reliée à la précédente par une relation rhétorique. Si dans (50) les informations rapportées paraissent à propos successivement de ce qui se passe en Allemagne et au Pakistan, c'est parce que le texte ne configure pas un récit (Ricoeur 1983-1985) et donc ne fait émerger aucun autre topique du discours (Marandin). La fonction cadrative des SP n'en demeure pas moins, son rôle est même plus important que dans (49) et elle est exploitée stratégiquement. Le texte suivant extrait du même corpus :

(51) "Dans un club de jazz, Greg Wonder, un saxophoniste de renom, dirige un excellent petit orchestre. Sur un terrain d'aviation, Eliot Vance virevolte aux commandes d'un ULM. Tous les deux suspendent leur activité à la demande de mystérieux émissaires qui leur font une offre d'un million de dollars à charge, pour eux, de les suivre à NewYork. Là, ils rencontrent un homme d'affaires ... ". (présentation du film Attention les dégâts)

illustre un cas proche, sauf que, à partir du moment où une intrigue se met en place, les SP de lieu ne constituent plus l'à propos du texte, ils passent au second plan, pour laisser le devant de la scène aux actions des personnages.

Relativement aux analyses de Jacobs rapportées dans la partie précédente, les observations qui précèdent plaident en faveur des deux idées suivantes :

\footnotetext{
${ }^{24} \mathrm{Cf}$. néanmoins pour un usage stratégique et systématique très frappant des conditionnelles l'étude de Marchese (1987) sur le godié.
} 
- a) tous les adverbiaux détachés en tête de phrase sont cadratifs dans le sens où ils sont à même de porter non seulement sur la proposition en tête de laquelle ils apparaissent mais sur une ou plusieurs autres figurant dans la suite. Les cadres qu'ils démimitent constituent des blocs informationnellement homogènes par rapport au critère signalé par l'adverbial. Ce critère fonctionne comme une sorte d'index que le lecteur ou l'auditeur doivent garder en mémoire pour le traitement de la phrase hôte de l'adverbial et au-delà, jusqu'à l'occurrence d'indices signalant que sa portée est terminée. Dans ce sens, qui n'est pas celui de Jacobs, tous les adverbiaux cadratifs ouvrent un fichier destiné à collationner les propositions rassemblées dans le cadre.

- b) les adverbiaux cadratifs ne sont en général pas adressés au sens de Jacobs. Les propositions qu'ils indexent ne portent pas sur le critère qu'ils spécifient. Les propositions qui tombent sous leur portée ne sont au sujet de l'index qu'ils mettent en avant que lorsque le discours ne fournit aucun autre topique à même de remplir cette fonction. Le fait que cette possibilité existe oblige à considérer qu'il n'est pas possible de statuer sur le topique (aboutness) d'une phrase isolée. Par défaut de contexte ultérieur, les adverbiaux détachés en tête de phrase, n'indiquent pas ce à propos de quoi est la phrase. Mais rien n'empêche que la suite oblige à leur restituer ce statut. Ce constat, s'il est bien fondé, milite en faveur d'approches dynamiques des phénomènes de topicalisation et conduit à réintégrer dans la discussion de ces phénomènes la notion de topique de discours (contra Reinhart 1982).

\section{Conclusion :}

Pour finir, nous aimerions attirer l'attention sur un aspect plus général. Depuis Halliday \& Hasan (1976) l'idée s'est plus ou moins imposée que les marques de cohésion discursive se répartissaient dans deux grands systèmes : l'un regroupant les marqueurs de continuité référentielle et l'autre les marqueurs de relations entre les contenus propositionnels et/ou les actes de langage. Reinhart (1981) note ainsi que: "The various devices for linking adjacent sentences in a discourse can be reduced to two types of link : the one is referential links between their expressions (...) The other type of cohesive link is a semantic link between the propositions expressed by the two sentences" et on retrouve la même idée (avec une terminologie légèrement différente) chez Sanders, Schilperoord \& Spooren qui, dans une publication récente (2001), parlent de cohérence et expliquent que celle-ci s'exprime au niveau "référentiel" et "relationnel". A propos de cette répartition, nous nous contenterons de faire remarquer que les relations référentielles exprimées notamment par les différentes formes d'anaphores et les relations "de discours" (ou "rhétoriques") entre contenus propositionnels et actes de langage exprimés par les connecteurs ont en commun d'être :

- asymétriques (elles impliquent respectivement un anaphorisé et un anaphorisant, un noyau et un satellite, autrement dit un constituant dépendant d'un autre)

- de fonctionner d'aval en amont (relations remontantes)

- et de porter sur deux unités adjacentes (simples ou complexes) et donc d'avoir une portée étroite.

Ces traits (qu'il faudrait préciser et nuancer) justifient, au plan général, qu'on les regroupe dans une même catégorie. Ce regroupement s'impose d'autant que ces relations de connexion (référentielle et rhétorique) s'opposent, globalement, aux relations d'indexation induites par des expressions adverbiales (SP, Adv, SN, sous-phrases adverbiales) détachées en tête de phrase (en position préverbale). Comme on l'a vu, ces expressions:

- sont non hiérarchisantes (les propositions rassemblées dans un même cadre forment une liste (elles sont toutes équivalentes en regard du critère exploité par l'introducteur de cadre), 
- fonctionnent d'amont en aval

- et sont potentiellement ouvertes en aval.

Si l'on veut bien accepter ces généralités, ne serait-ce qu'à titre d'hypothèse de travail, on voit immédiatement que le fait que les adverbiaux antéposés jouissent d'un potentiel cadratif revêt une importance particulière dans la mesure où ces expressions, jusque-là non répertoriées dans les taxinomies de relations cohésives, constituent une des deux grandes familles de marques disponibles dans les différentes langues pour guider le lecteur dans l'accès à la cohérence du discours. Les adverbiaux cadratifs jouent un rôle original dans ce guidage : quand ils ne sont pas liés par la postposition du sujet de la proposition en tête de laquelle ils apparaissent (cf. C.Fuchs \& N.Fournier, ce volume), ils servent à la répartition des informations textuelles en fonction des circonstances attachées aux états de choses communiqués ou à leur énonciation. Ces index ont pour vocation d'être adressés en mémoire et de faciliter la récupération des fait(s) mentionné(s) et/ou du ou des énoncé(s) y faisant allusion $^{25}$. Dans certains cas bien particuliers, ils peuvent fonctionner comme des topiques au sens d"'aboutness", mais en général, les segments qu'ils préfixent sont à propos d'autre chose.

Le fait que les adverbiaux antéposés jouissent de ce pouvoir d'indexation est lié à la place qu'ils occupent, i.e. au fait qu'ils précèdent la mise en place de la prédication. Comme cette place est pragmatiquement destinée à accueillir :

- des informations déjà évoquées, les adverbiaux cadratifs entretiennent des liens privilégiées avec les différentes formes d'anaphore,

- des marqueurs de relation rhétoriques, ils cumulent aussi très facilement des fonctions de connecteurs.

Les deux grands systèmes de relations qui interviennent dans la cohésion extraphrastique sont donc étroitement liés, sauf qu'ils tirent dans deux directions opposées : les anaphores et les connecteurs tirent vers l'amont, les adverbiaux cadratifs vers l'aval, avec, bien entendu, toutes sortes de formes qui tirent dans les deux sens, sans compter celles qui, dans l'histoire, ont fini par tirer à gauche alors qu'elles tiraient plutôt à droite (cf. mais et et qui, avant de devenir de purs connecteurs étaient des adverbiaux), l'inverse semblant plus rare.

\section{Bibliographie :}

BERTHONNEAU A. M., 1987, "La thématisation et les compléments antéposés", Travaux de Linguistique, 14-15.

CHAFE W., 1976, "Giveneness, contrastiveness, subjects, topics, and point of view", in C.Li ed. Subject and topic, New-York, Academis Press.

CHAFE W., 1984, "How people use adverbial clauses", in C.Brugman \& M.Macaulay eds., Proceedings of the Tenth Annual Meeting of the Berkeley Linguistics Society, Berkeley, Berkeley Linguistics Society, 437-459.

CHAFE W., 1994, Discourse, Consciousness and Time. The Flow and Displacement of Conscious Experience in Speaking and Writing, Chicago, Chicago University Press.

CHAROLlES M., 1995, "Cohésion, cohérence et pertinence du discours", Travaux de Linguistique, 29, 125-151.

CHAROLLES M., 1997, L'encadrement du discours : univers, champs, domaines et espaces, Cahier de Recherche Linguistique, LANDISCO, URA-CNRS 1035 Université Nancy 2, n 6, 1-73. Accessible sur le site http://www.ltm.ens.fr/siteACFT/

\footnotetext{
${ }^{25}$ Des expérimentations visant à tester ces conjectures psycholinguistiques sont en cours en collaboration avec J.Pynte et S.Colonna (Aix en Provence).
} 
CHAROLLES M \& LAMIROY B. 2002, "Syntaxe phrastique et transphrastique : du but au résultat", in H.Nolke \& H.L. Andersen eds. Macrosyntaxe et macrosémantique, Actes du colloque international dAarhus, 17-19 mai 2001, Bern, Peter Lang, 383-419.

COMBETTES B., 1998, Les constructions détachées en français, Ophrys, 143 p.

DIESSEL, H., 2001, "The ordering distribution of main and adverbial clauses : a typological study". Language: $77,2,433-453$.

DUCROT O., 1972, Dire et ne pas dire, Paris, Hermann. (chap. 6)

ENKVIST N., 1987, "Text strategies : single, dual, multiple", in R.Steele \& T. Threadgold eds. Language, Topics. Essays in Honour of Michael Halliday, Vol. II, Amsterdam, John Benjamins, 203-211.

FORD C; \& THOMPSON S., 1986, "Conditionals in Discourse : A Text based study from English", in E.Traugott, C.Ferguson, J.Reilly \& A. ter Meulen éds, On Conditionals, Cambridge, CUP.

FRANCKEL J.J. \& PAILLARD D., 1999, "Considérations sur l'antéposition des syntagmes prépositionnels", in C.Guimier ed. La thématisation dans les langues, Bern, Peter Lang, 277-295.

GUIMIER C., ed, 1993, 1001 circonstants, Caen, PUC.

GUIMIER C., 1996, Les adverbes du français. Le cas des adverbes en "ment", Paris Ophrys.

HAIMAN J., 1978, "Conditionals are topics", Language, 54, 564-589.

HALLIDAY M.A.K. \& HASAN R., 1976, Cohesion in English, London, Longman.

HASSELGARD H., à par. a, The role of multiple themes in cohesion". To appear in K. Aijmer, \& A.B. Brita Stenstöm (eds): Discourse Patterns in Spoken and Written Corpora. Benjamins.

HASSELGARD H.,à par.b, "Temporal and spatial adjuncts as elements of texture". To appear in D.Banks (ed.). Proceedings from the $14^{\text {th }}$ Euro-International Systemic Functional Workshop. Paris: L'Harmattan.

HUUMO T., 1996, "A scoping hierarchy of locatives", Cognitive Linguistics, 7, 3, 265-299.

JACOBS, J., 2001, "The dimension of topic-comment", Linguistics, 39-4, 641-681.

JESPERSEN O., 1940, A Modern English Grammar on historical principles, V Syntax, London, George Allen \& Unwin.

LAMBRECHT, K., 1994, Information Structure and Sentence Form. Topic, focus, and the mental representation of discourse referents, Cambridge, Cambridge University Press.

LAMBRECHT K., à par.a, "Dislocation" in M.Haspelmath and alii, eds., Language Typology and Language Universals, Berlin and New-York: de Gruyter.

LAMBRECHT K., à par.b, "On the interaction of information structure and formal structure in constructions. The case of French Right-Detached comme N", in J.O.Ostman \& M.Fried eds., Construction Grammar(s): Cognitive and Cross-Language Dimensions, Amsterdam, Benjamins.

LEEMAN D., 1998, Les circonstants en question(s), Paris, Kimé.

LE DRAOULEC A. \& PERY-WOODLEY M.P., à par., "Time Travel in Text : Temporal Framing in Narratives and Non-Narratives"

LE GOFFIC P., 1993, Grammaire de la phrase française, Paris, Hachette.

LE QUERLER N., 1993, "Les circonstants en position intiale", in C.Guimier ed., 1001 circonstants, Caen, PUC, 159-184.

MARCHESE, L., 1987, "On the role of conditionnals in Godié. Procedural discourse", in R.S.Tomlin ed. Coherence and Grounding in discourse. Amsterdam, John Benjamins.

MARANDIN J.M., 1988, "A propos de la notion de topique de discours. Eléments d'analyse dans le récit", Langue Française, 78, 67-88.

MELIS L., 1983, Les Circonstants et la phrase. Etude sur la classification et la systématique des compléments circonstanciels en français moderne, Leuven, Presses Universitaires. 
MOLINIER, C. \& LEVRIER F., 2000, Grammaire des adverbes, Description des formes en ment, Genève-Paris, Droz.

PREVOST S., à par., "Détachement et topicalisation : des niveaux d'analyse différents"

RAMSAY V., 1984, "Preposed and postposed "if" and "when" clauses", in R.Tomlin ed., Coherence and Grounding in Discourse, Amsterdam, Benjamins, 383-408.

REINHART, T., 1981, "Pragmatics and linguistics. An analysis of sentence topics", Philosophica, 27, 53-94.

RICOEUR P, 1983, 1984, 1985, Temps et récit, Paris, Seuil.

ROSSI M., 1999, L'intonation, le système du français. Description et modélisation, Paris, Ophrys.

SANDERS T., SCHILPEROORD J. \& SPOOREN W., eds, 2001, Text Representation: Linguistic and Psycholinguistic Aspects, Amsterdam, Benjamins.

SCHREPFER-ANDRE,G., à par., "Sur la portée textuelle des introducteurs de cadres de discours en selon X : les indices de fermeture des universénonciatifs", Actes du Colloque international "La Médiation, marquage en langue et en discours" (Université de Rouen, décembre 2000).

SERAFIN V., 1993, "Les circonstants en position finale", in C.Guimier ed., 1001 circonstants, Caen, PUC, 185-206.

TERRAN, E., 2002 : Le cadrage temporel en français, Thèse de Doctorat, Université de Paris III.

VIGIER, D., à par., "Les syntagmes prépositionnels en «en $N$ » détachés en tête de phrase référant à des domaines d'activité", Actes du Colloque Syntaxe et Sémantique (Bari, septembre 2002), Linguisticae Investigationes.

VIRTANEN T., 1992, "Temporal adverbials in text structuring : On temporal text strategiy", in A.C.Lindberg, N.Enkvist \& K.Wikberg eds., Nordic Research on Text and Discourse: NORDTEXT symposium 1990, Abo, Abo Academic Press, 185-197.

VOGUE DE S., 1999, "Le champ des subordonnées dites conditionnelles du français : conditions, éventualités, suppositions et hypothèses", LINX, 41, 93-118. 